\title{
Synthesis of the Tetracyclic Core of the Kempanes by a Ring-Closing Metathesis Strategy
}

\author{
Liang Zhao and D. Jean Burnell* \\ Department of Chemistry, Dalhousie University, Halifax NS, Canada B3H 4J3 \\ jean.burnell@dal.ca
}

Table of Contents

Procedures and spectral data for $\mathbf{1 4}$ and $\mathbf{1 5}$

S2

Procedure and spectral data for $\mathbf{1 6}$

S4

Procedures and spectral data for $\mathbf{1 7}$ and $\mathbf{1 8}$

S5

Procedures and spectral data for $\mathbf{1 9}$ and $\mathbf{2 0}$

S7

Procedure and spectral data for 22a and 22b

S8

Procedure and spectral data for $\mathbf{2 3}$

S9

Procedure and spectral data for $\mathbf{2 4}$

S10

${ }^{1} \mathrm{H}$ and ${ }^{13} \mathrm{C}$ NMR spectra

$\mathrm{S} 11-\mathrm{S} 20$ 


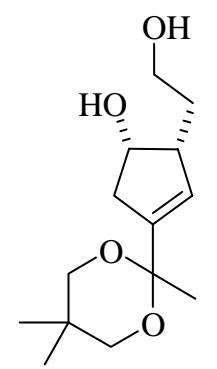

$\mathrm{LiAlH}_{4}(97 \mathrm{mg}, 2.5 \mathrm{mmol})$ was added to a solution of $12(314 \mathrm{mg}, 1.25 \mathrm{mmol})$ in dry THF $(20 \mathrm{~mL})$ at 0 ${ }^{\circ} \mathrm{C}$. The mixture was stirred at $\mathrm{rt}$ for $15 \mathrm{~h}$. A small amount of $\mathrm{H}_{2} \mathrm{O}$ and then of $15 \%$ aqueous $\mathrm{NaOH}$ were added, followed by more $\mathrm{H}_{2} \mathrm{O}$. Ether was added, and the ether solution was washed with brine and dried $\left(\mathrm{MgSO}_{4}\right)$. Evaporation of the solvent left an oily residue, which was purified by flash chromatography (here and elsewhere: 230-400 mesh silica gel, eluted with hexanes containing an increasing proportion of ethyl acetate) to provide 14 (297 mg, 93\%) as a liquid: IR (neat): 3384, 1641 $\mathrm{cm}^{-1} ;{ }^{1} \mathrm{H}$ NMR $\left(500 \mathrm{MHz}, \mathrm{CDCl}_{3}\right): \delta 5.57(1 \mathrm{H}$, br s), $4.51(1 \mathrm{H}, \mathrm{m}), 3.80(1 \mathrm{H}, \mathrm{m}), 3.74(2 \mathrm{H}, \mathrm{br} \mathrm{OH})$, $3.68(1 \mathrm{H}, \mathrm{m}), 3.54(2 \mathrm{H}, \mathrm{m}), 3.35(2 \mathrm{H}, \mathrm{d}, J=11.0 \mathrm{~Hz}), 2.87(1 \mathrm{H}, \mathrm{m}), 2.61(1 \mathrm{H}, \mathrm{dd}, J=6.0,17.5 \mathrm{~Hz})$, $2.32(1 \mathrm{H}, \mathrm{brd}, J=17.5 \mathrm{~Hz}), 1.91(1 \mathrm{H}, \mathrm{m}), 1.78(1 \mathrm{H}, \mathrm{m}), 1.43(3 \mathrm{H}, \mathrm{s}), 1.16(3 \mathrm{H}, \mathrm{s}), 0.72(3 \mathrm{H}, \mathrm{s}) ;{ }^{13} \mathrm{C}$ $\mathrm{NMR}\left(125 \mathrm{MHz}, \mathrm{CDCl}_{3}\right.$, here and elsewhere: in parentheses, the numbers of attached hydrogens are based on DEPT): $\delta 140.9$ (0), 130.9 (1), 98.6 (0), 72.5 (1), 71.9 (2), 71.8 (2), 61.6 (2), 49.5 (1), 41.2 (2), 30.5 (2), $29.8(0), 27.2(3), 22.8$ (3), $22.2(3)$; MS: $m / z 256\left(\mathrm{M}^{+}\right)$.

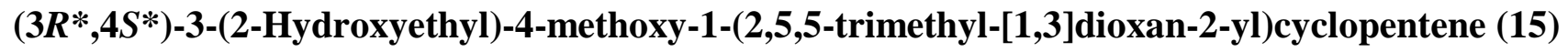

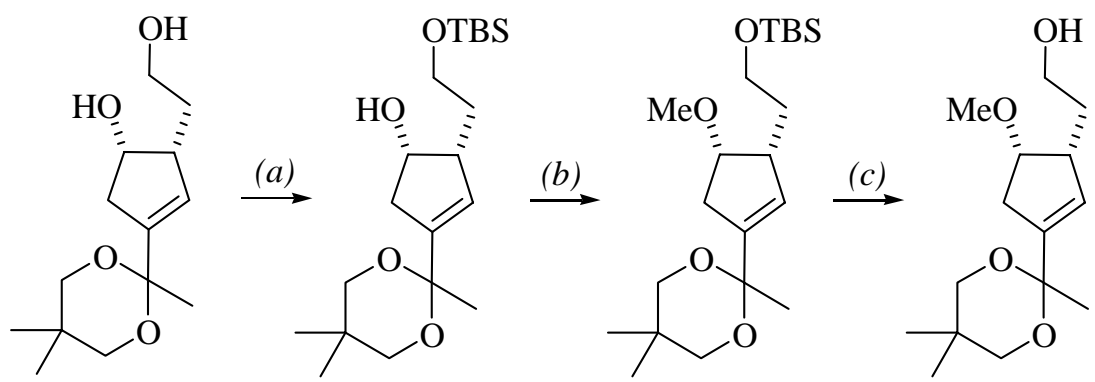

(a) A solution of tert-butylchlorodimethylsilane $(175 \mathrm{mg}, 1.16 \mathrm{mmol})$ in $\mathrm{CH}_{2} \mathrm{Cl}_{2}(20 \mathrm{~mL})$ was added dropwise at $\mathrm{rt}$ to a $\mathrm{CH}_{2} \mathrm{Cl}_{2}(20 \mathrm{~mL})$ solution of imidazole $(237 \mathrm{mg}, 3.48 \mathrm{mmol})$ and $14(297 \mathrm{mg}, 1.16$ mmol). This was stirred at rt for $15 \mathrm{~h}$. The solution was washed with water, sat. $\mathrm{NaHCO}_{3}$ and brine, dried $\left(\mathrm{Na}_{2} \mathrm{SO}_{4}\right)$ and concentrated under vacuum. Flash chromatography gave the monosilylated product (402 mg, 94\%) as an oil: ${ }^{1} \mathrm{H}$ NMR (500 MHz, $\left.\mathrm{CDCl}_{3}\right): \delta 5.51(1 \mathrm{H}$, br s), $4.48(1 \mathrm{H}, \mathrm{m}), 3.83(1 \mathrm{H}, \mathrm{m})$, 
$3.66(1 \mathrm{H}$, br t, $J=8.8 \mathrm{~Hz}), 3.57(1 \mathrm{H}, \mathrm{d}, J=11.0 \mathrm{~Hz}), 3.55(1 \mathrm{H}, \mathrm{d}, J=11.0 \mathrm{~Hz}), 3.51(1 \mathrm{H}, \mathrm{OH}), 3.33$ $(2 \mathrm{H}, \mathrm{d}, J=11.0 \mathrm{~Hz}), 2.82(1 \mathrm{H}, \mathrm{m}), 2.61(1 \mathrm{H}, \mathrm{br} \mathrm{d}, J=16.8 \mathrm{~Hz}), 2.34(1 \mathrm{H}, \mathrm{d}, J=16.8 \mathrm{~Hz}), 1.95(1 \mathrm{H}$, m), $1.77(1 \mathrm{H}, \mathrm{d}, J=11.0 \mathrm{~Hz}), 1.43(3 \mathrm{H}, \mathrm{s}), 1.17(3 \mathrm{H}, \mathrm{s}), 0.93(9 \mathrm{H}, \mathrm{s}), 0.70(3 \mathrm{H}, \mathrm{s}), 0.11(3 \mathrm{H}, \mathrm{s}), 0.10$ $(3 \mathrm{H}, \mathrm{s}) .(b)$ To a suspension of $\mathrm{NaH}(60 \%$ in mineral oil, $51 \mathrm{mg}, 1.27 \mathrm{mmol})$ in $\mathrm{THF}(10 \mathrm{~mL})$ at $0{ }^{\circ} \mathrm{C}$ was added dropwise a solution of the monosilylated product of (a) (235 mg, $0.635 \mathrm{mmol})$ in THF (10 $\mathrm{mL}$ ), followed by HMPA (342 mg, $1.91 \mathrm{mmol}) . \mathrm{CH}_{3} \mathrm{I}(270 \mathrm{mg}, 1.91 \mathrm{mmol})$ was added, and the solution was stirred for $15 \mathrm{~h}$ at $\mathrm{rt} . \mathrm{H}_{2} \mathrm{O}$ was added, and the solvent was evaporated under vacuum. $\mathrm{H}_{2} \mathrm{O}$ and EtOAc were added. The EtOAc extract was washed with $\mathrm{H}_{2} \mathrm{O}$ and brine, and the solvent was removed under vacuum. Flash chromatography of the residue gave the methylated product (221 mg, 91\%) as a liquid: ${ }^{1} \mathrm{H} \mathrm{NMR}\left(500 \mathrm{MHz}, \mathrm{CDCl}_{3}\right): \delta 5.74(1 \mathrm{H}, \mathrm{br} \mathrm{s}), 3.98(1 \mathrm{H}$, apparent q, $J=6.2 \mathrm{~Hz}), 3.70(2 \mathrm{H}, \mathrm{m})$, $3.52(2 \mathrm{H}, \mathrm{m}), 3.33(2 \mathrm{H}$, overlapped signal), $3.32(3 \mathrm{H}, \mathrm{s}), 2.89(1 \mathrm{H}, \mathrm{m}), 2.43(1 \mathrm{H}, \mathrm{dd}, J=6.3,16.1 \mathrm{~Hz})$, $2.32(1 \mathrm{H}, \mathrm{dd}, J=5.0,16.1 \mathrm{~Hz}), 1.87(1 \mathrm{H}, \mathrm{m}), 1.55(1 \mathrm{H}, \mathrm{m}), 1.40(3 \mathrm{H}, \mathrm{s}), 1.17(3 \mathrm{H}, \mathrm{s}), 0.90(3 \mathrm{H}, \mathrm{s})$, $0.70(3 \mathrm{H}, \mathrm{s}), 0.06(6 \mathrm{H}, \mathrm{s})$.

(c) Solid TBAF (752 mg, $2.88 \mathrm{mmol})$ was added to a solution of the methylated product of $(b)(221$ $\mathrm{mg}, 0.576 \mathrm{mmol})$ in THF (20 mL) at rt. The mixture was stirred for $15 \mathrm{~h}$ before $\mathrm{H}_{2} \mathrm{O}$ was added. The solvent was removed under vacuum, and the residue was taken up in EtOAc. The combined solutions were washed with $\mathrm{H}_{2} \mathrm{O}$ and brine, dried $\left(\mathrm{Na}_{2} \mathrm{SO}_{4}\right)$, and concentrated under vacuum. Flash chromatography gave 15 (155 mg, 100\%) as a liquid: IR (neat): 3438, $1640 \mathrm{~cm}^{-1} ;{ }^{1} \mathrm{H}$ NMR $(500 \mathrm{MHz}$, $\left.\mathrm{CDCl}_{3}\right): \delta 5.61(1 \mathrm{H}$, br s), $4.01(1 \mathrm{H}$, apparent q, $J=6.8 \mathrm{~Hz}), 3.54-3.65(2 \mathrm{H}, \mathrm{m}), 3.44(2 \mathrm{H}, \mathrm{m}), 3.28$ $(3 \mathrm{H}, \mathrm{s}), 3.26(2 \mathrm{H}$, overlapped m), $3.04(1 \mathrm{H}$, br OH$), 2.90(1 \mathrm{H}, \mathrm{m}), 2.40(1 \mathrm{H}, \mathrm{dd}, J=7.0,16.5 \mathrm{~Hz}), 2.29$ $(1 \mathrm{H}$, br dd, $J=5.5,16.5 \mathrm{~Hz}), 1.82(1 \mathrm{H}, \mathrm{m}), 1.61(1 \mathrm{H}, \mathrm{m}), 1.33(3 \mathrm{H}, \mathrm{s}), 1.09(3 \mathrm{H}, \mathrm{s}), 0.64(3 \mathrm{H}, \mathrm{s}) ;{ }^{13} \mathrm{C}$ NMR (125 MHz, $\mathrm{CDCl}_{3}$ ): $\delta 140.2$ (0), 131.2 (1), 98.4 (0), 82.0 (1), 71.7 (2C, 2), 61.3 (2), 57.1 (3), 46.0 (1), 36.3 (2), 31.2 (2), 29.7 (0), 27.3 (3), 22.7 (3), 22.1 (3); MS: $m / z 270.1824\left(\mathrm{M}^{+}\right)$, required for $\mathrm{C}_{15} \mathrm{H}_{26} \mathrm{O}_{4}: 270.1831$. 


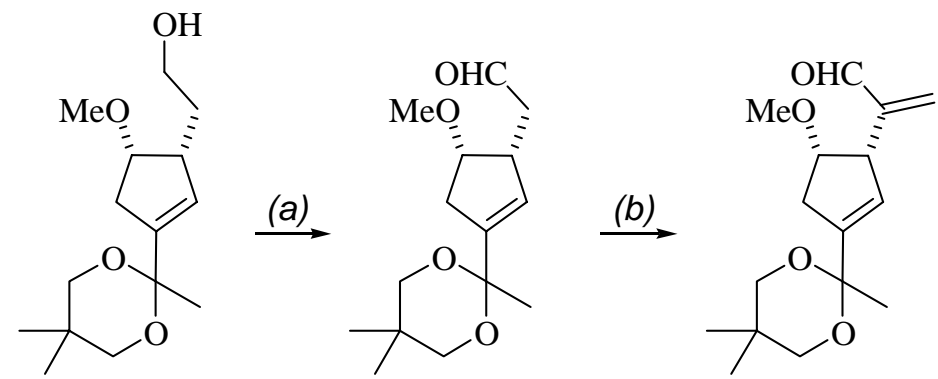

(a) Dess-Martin periodinane $(2.87 \mathrm{mg}, 6.76 \mathrm{mmol})$ was added to a solution of $\mathbf{1 5}$ (1.52 $\mathrm{mg}, 5.64$ mmol) and $\mathrm{NaHCO}_{3}(5.0 \mathrm{~g})$ in $\mathrm{CH}_{2} \mathrm{Cl}_{2}(200 \mathrm{~mL})$ at rt. The mixture was stirred at $\mathrm{rt}$ for $12 \mathrm{~h} . \mathrm{H}_{2} \mathrm{O}$ was added. The $\mathrm{CH}_{2} \mathrm{Cl}_{2}$ layer was washed with $10 \%$ aqueous $\mathrm{Na}_{2} \mathrm{~S}_{2} \mathrm{O}_{3}$, sat. $\mathrm{NaHCO}_{3}$, and brine. The solution was dried $\left(\mathrm{Na}_{2} \mathrm{SO}_{4}\right)$ and concentrated under vacuum. GC-MS analysis of the liquid residue indicated complete oxidation to the aldehyde, which was used immediately in the next step, but when a small, retained sample was analyzed by NMR it was evident that the product contained $40 \%$ unoxidized 15. Data for the aldehyde (from the $3: 2$ mixture of aldehyde and 15): ${ }^{1} \mathrm{H} \mathrm{NMR}\left(500 \mathrm{MHz}, \mathrm{CDCl}_{3}\right): \delta$ $9.73(1 \mathrm{H}, \mathrm{s}), 5.59(1 \mathrm{H}, \mathrm{br} \mathrm{s}), 4.00(1 \mathrm{H}, \mathrm{m}), 3.42(2 \mathrm{H}, \mathrm{m}), 3.26(2 \mathrm{H}, \mathrm{m}), 3.21(3 \mathrm{H}, \mathrm{s}), 2.91(1 \mathrm{H}, \mathrm{m}), 2.64$ (1H, ddd, $J=1.8,8.3,17.0 \mathrm{~Hz}), 2.29-2.46(2 \mathrm{H}, \mathrm{m}), 2.27(1 \mathrm{H}, \mathrm{m}), 1.32(3 \mathrm{H}, \mathrm{s}), 1.08(3 \mathrm{H}, \mathrm{s}), 0.64(3 \mathrm{H}$, s); ${ }^{13} \mathrm{C} \mathrm{NMR} \mathrm{(125} \mathrm{MHz,} \mathrm{CDCl}_{3}$ ): $\delta 201.9$ (1), 141.5 (0), 129.9 (1), 98.2 (0), 81.2 (1), 71.7 (2), 71.6 (2), 57.2 (3), 43.7 (1), 42.7 (2), 36.7 (2), 29.7 (0), 27.2 (3), 22.6 (3), 22.1 (3); MS: m/z $268.1671\left(\mathrm{M}^{+}\right)$, required for $\mathrm{C}_{15} \mathrm{H}_{24} \mathrm{O}_{4}: 268.1674$.

(b) Methylenedimethylammonium iodide $(2.09 \mathrm{~g}, 11.3 \mathrm{mmol})$ was added to a solution of the crude aldehyde from (a) (presumed $5.64 \mathrm{mmol}$ ) and $\mathrm{Et}_{3} \mathrm{~N}(5.7 \mathrm{~g}, 56 \mathrm{mmol})$ in $\mathrm{CH}_{2} \mathrm{Cl}_{2}(50 \mathrm{~mL})$, and the mixture was stirred for $14 \mathrm{~h}$ at rt. The mixture washed with sat. $\mathrm{NaHCO}_{3}$ and brine. The organic layer was dried $\left(\mathrm{Na}_{2} \mathrm{SO}_{4}\right)$ and concentrated under vacuum. Flash chromatography gave $16(0.947 \mathrm{~g}, 60 \%$ from 15, but $100 \%$ based on the proportion of the aldehyde in the starting material) as an oil: IR (neat): 2698, $1692 \mathrm{~cm}^{-1} ;{ }^{1} \mathrm{H}$ NMR $\left(500 \mathrm{MHz}, \mathrm{CDCl}_{3}\right): \delta 9.62(1 \mathrm{H}, \mathrm{s}), 6.22(1 \mathrm{H}, \mathrm{s}), 6.13(1 \mathrm{H}, \mathrm{s}), 5.67(1 \mathrm{H}, \mathrm{br}$ s), $4.23(1 \mathrm{H}, \mathrm{m}), 4.04(1 \mathrm{H}, \mathrm{br} \mathrm{d}, J=7.5 \mathrm{~Hz}), 3.55(2 \mathrm{H}, \mathrm{m}), 3.38(2 \mathrm{H}, \mathrm{m}), 3.19(3 \mathrm{H}, \mathrm{s}), 2.55(1 \mathrm{H}, \mathrm{dd}, J$ $=6.2,17.1 \mathrm{~Hz}), 2.39(1 \mathrm{H}, \mathrm{br} \mathrm{d}, J=17.1 \mathrm{~Hz}), 1.46(3 \mathrm{H}, \mathrm{s}), 1.18(3 \mathrm{H}, \mathrm{s}), 0.73(3 \mathrm{H}, \mathrm{s}) ;{ }^{13} \mathrm{C}$ NMR $(125$ $\mathrm{MHz}, \mathrm{CDCl}_{3}$ ): $\delta 194.3$ (1), 147.9 (0), 143.8 (0), 134.3 (2), 127.9 (1), 98.5 (0), 81.5 (1), 72.0 (2), 71.9 (2), 57.7 (3), 47.2 (1), 37.6 (2), 29.9 (0), 27.6 (3), 22.8 (3), 22.3 (3); MS: m/z 280.1688 (M+), required for $\mathrm{C}_{16} \mathrm{H}_{24} \mathrm{O}_{4}: 280.1674$. 


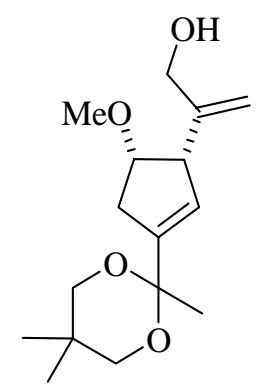

$\mathrm{NaBH}_{4}(255 \mathrm{mg}, 6.75 \mathrm{mmol})$ was added to a solution of $16(1.89 \mathrm{~g}, 6.75 \mathrm{mmol})$ and $\mathrm{CeCl}_{3} \cdot 7 \mathrm{H}_{2} \mathrm{O}(2.52$ g, $6.75 \mathrm{mmol})$ in $\mathrm{EtOH}(100 \mathrm{~mL})$ at $0{ }^{\circ} \mathrm{C}$. EtOAc and $\mathrm{H}_{2} \mathrm{O}$ were added when TLC showed consumption of 16 was complete. The EtOAc layer was washed with brine and dried $\left(\mathrm{Na}_{2} \mathrm{SO}_{4}\right)$ giving $17(1.73 \mathrm{~g}$, 91\%) as an oil: ${ }^{1} \mathrm{H}$ NMR (500 MHz, $\left.\mathrm{CDCl}_{3}\right): \delta 5.64(1 \mathrm{H}$, br s), $5.21(1 \mathrm{H}, \mathrm{s}), 5.02(1 \mathrm{H}, \mathrm{s}), 4.23(1 \mathrm{H}, \mathrm{m})$, $4.14(1 \mathrm{H}, \mathrm{dd}, J=2.5,12.6 \mathrm{~Hz}), 4.06(1 \mathrm{H}, \mathrm{dd}, J=4.8,12.6 \mathrm{~Hz}), 3.71(1 \mathrm{H}, \mathrm{br} \mathrm{d}, J=7.5 \mathrm{~Hz}), 3.60(1 \mathrm{H}$, $\mathrm{d}, J=11.0 \mathrm{~Hz}), 3.52(1 \mathrm{H}, \mathrm{d}, J=11.3 \mathrm{~Hz}), 3.34-3.39(2 \mathrm{H}$, overlapped signal), $3.36(3 \mathrm{H}, \mathrm{s}), 3.22(1 \mathrm{H}$, $\mathrm{OH}), 2.54(1 \mathrm{H}, \mathrm{dd}, J=7.0,16.5 \mathrm{~Hz}), 2.40(1 \mathrm{H}, \mathrm{br} \mathrm{dd}, J=6.0,16.5 \mathrm{~Hz}), 1.45(3 \mathrm{H}, \mathrm{s}), 1.18(3 \mathrm{H}, \mathrm{s}), 0.72$ $(3 \mathrm{H}, \mathrm{s}) ;{ }^{13} \mathrm{C}$ NMR (125 MHz, $\mathrm{CDCl}_{3}$ ): $\delta 146.5$ (0), 142.5 (0), 129.4 (1), 114.9 (2), 98.5 (0), 82.7 (1), 72.0 (2), 71.9 (2), 66.0 (2), 57.6 (3), 53.3 (1), 36.7 (2), 29.8 (0), 27.6 (3), 22.8 (3), 22.3 (3); MS: m/z 282.1826 $\left(\mathrm{M}^{+}\right)$, required for $\mathrm{C}_{16} \mathrm{H}_{26} \mathrm{O}_{4}: 282.1831$.

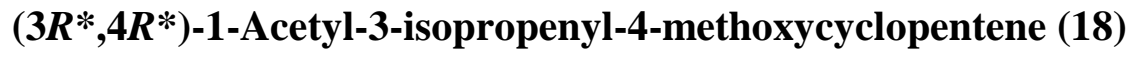

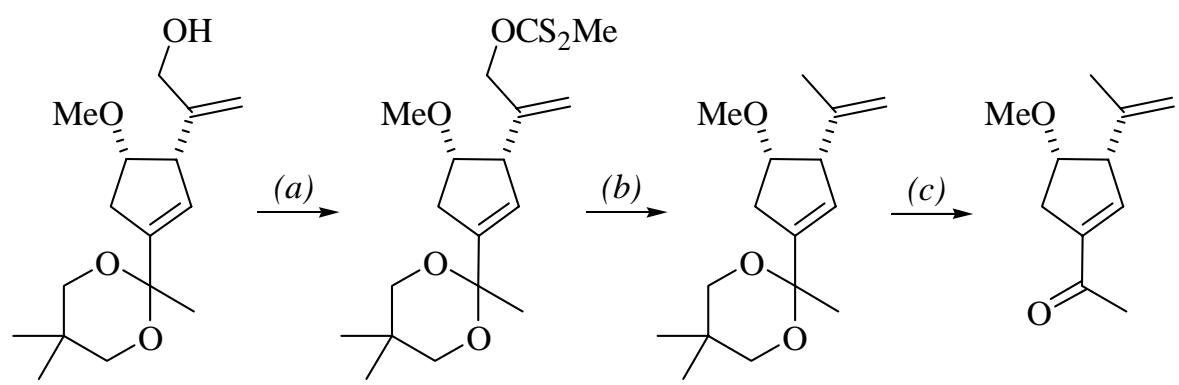

(a) To a suspension of $\mathrm{NaH}(60 \%$ in mineral oil, $477 \mathrm{mg}, 11.9 \mathrm{mmol})$ in $\mathrm{THF}(20 \mathrm{~mL})$ at $0{ }^{\circ} \mathrm{C}$ was added a solution of $17(672 \mathrm{mg}, 2.38 \mathrm{mmol})$ in THF (5 mL). The mixture was allowed to warm to rt, and it was stirred at $\mathrm{rt}$ for $30 \mathrm{~min}$. The mixture was cooled to $0{ }^{\circ} \mathrm{C}$, and $\mathrm{CS}_{2}(0.90 \mathrm{~g}, 12 \mathrm{mmol})$ was added. The mixture was allowed to warm to $\mathrm{rt}$, and it was stirred at $\mathrm{rt}$ for $1 \mathrm{~h}$. The mixture was cooled once more to $0{ }^{\circ} \mathrm{C}$, and more $\mathrm{CS}_{2}(0.90 \mathrm{~g}, 12 \mathrm{mmol})$ was added. The mixture was warmed to rt, $\mathrm{CH}_{3} \mathrm{I}$ 
(1.69 $\mathrm{g}, 0.74 \mathrm{~mL}, 11.9 \mathrm{mmol})$ was added, and the mixture was stirred at $\mathrm{rt}$ for $14 \mathrm{~h} . \mathrm{H}_{2} \mathrm{O}(5 \mathrm{~mL})$ was added, and the volatile organics were removed under vacuum. The residue was extracted with $\mathrm{CH}_{2} \mathrm{Cl}_{2}$. The combined $\mathrm{CH}_{2} \mathrm{Cl}_{2}$ layers were washed with $\mathrm{H}_{2} \mathrm{O}$ and brine the dried $\left(\mathrm{Na}_{2} \mathrm{SO}_{4}\right)$. The solvent was removed under vacuum, and flash chromatography of the residue yielded the thiocarbonate $(680 \mathrm{mg}$, $77 \%$ ) as an oil: IR (neat): $1671,1649 \mathrm{~cm}^{-1} ;{ }^{1} \mathrm{H}$ NMR (500 MHz, $\left.\mathrm{CDCl}_{3}\right): \delta 5.71(1 \mathrm{H}$, br s), $5.28(1 \mathrm{H}$, s), $5.21(1 \mathrm{H}, \mathrm{d}, J=13.6 \mathrm{~Hz}), 5.13(1 \mathrm{H}, \mathrm{s}), 5.12(1 \mathrm{H}, \mathrm{d}, J=13.6 \mathrm{~Hz}), 4.17(1 \mathrm{H}, \mathrm{m}), 3.66(1 \mathrm{H}, \mathrm{br} \mathrm{d}, J=$ $7.0 \mathrm{~Hz}), 3.59(1 \mathrm{H}, \mathrm{d}, J=11.0 \mathrm{~Hz}), 3.51(1 \mathrm{H}, \mathrm{d}, J=10.5 \mathrm{~Hz}), 3.36(2 \mathrm{H}, \mathrm{m}), 3.32(3 \mathrm{H}, \mathrm{s}), 2.58(3 \mathrm{H}, \mathrm{s})$, $2.53(1 \mathrm{H}, \mathrm{dd}, J=6.8,16.7 \mathrm{~Hz}), 2.38(1 \mathrm{H}, \mathrm{br} \mathrm{dd}, J=5.0,16.7 \mathrm{~Hz}), 1.44(3 \mathrm{H}, \mathrm{s}), 1.18(3 \mathrm{H}, \mathrm{s}), 0.71(3 \mathrm{H}$, s); ${ }^{13} \mathrm{C}$ NMR (125 MHz, $\mathrm{CDCl}_{3}$ ): $\delta 215.8$ (0), 143.1 (0), $141.6(0), 128.8$ (1), $115.4(2), 98.6$ (0), 82.2 (1), 75.9 (2), 72.0 (2C, 2), 57.7 (3), 52.9 (1), 37.3 (2), 29.9 (0), 27.7 (3), 22.9 (3) and 22.3 (3); MS: $\mathrm{m} / \mathrm{z}$ $372.1418\left(\mathrm{M}^{+}\right)$, required for $\mathrm{C}_{18} \mathrm{H}_{28} \mathrm{O}_{4} \mathrm{~S}_{2}: 372.1429$.

(b) To a solution of the thiocarbonate product from (a) (480 $\mathrm{mg}, 1.29 \mathrm{mmol})$ in deoxygenated benzene $(25 \mathrm{~mL})$ was added AIBN (42 mg, $0.25 \mathrm{mmol})$ and $n-\mathrm{Bu}_{3} \mathrm{SnH}(0.75 \mathrm{~g}, 0.70 \mathrm{~mL}, 2.6 \mathrm{mmol})$ at $\mathrm{rt}$. The mixture was heated at reflux for $12 \mathrm{~h}$. The mixture was cooled to $\mathrm{rt}$, and the solvent was evaporated under vacuum. Flash chromatography of the residue provided the reduced compound (12) (329 mg, 96\%) as a liquid: ${ }^{1} \mathrm{H}$ NMR $\left(500 \mathrm{MHz}, \mathrm{CDCl}_{3}\right): \delta 5.65(1 \mathrm{H}$, narrow $\mathrm{m}), 4.88(1 \mathrm{H}$, br s), $4.82(1 \mathrm{H}$, br s), $4.16(1 \mathrm{H}, \mathrm{m}), 3.60(1 \mathrm{H}, \mathrm{d}, J=11.0 \mathrm{~Hz}), 3.52(1 \mathrm{H}, \mathrm{d}, J=11.0 \mathrm{~Hz}), 3.51(1 \mathrm{H}, \mathrm{m}), 3.36(2 \mathrm{H}$, overlapped m), $3.34(3 \mathrm{H}, \mathrm{s}), 2.51(1 \mathrm{H}, \mathrm{dd}, J=7.2,16.0 \mathrm{~Hz}), 2.38(1 \mathrm{H}, \mathrm{dd}, J=6.0,16.0 \mathrm{~Hz}), 1.81(3 \mathrm{H}, \mathrm{s}), 1.44$ $(3 \mathrm{H}, \mathrm{s}), 1.18(3 \mathrm{H}, \mathrm{s}), 0.70(3 \mathrm{H}, \mathrm{s}) ;{ }^{13} \mathrm{C} \mathrm{NMR}\left(125 \mathrm{MHz}, \mathrm{CDCl}_{3}\right): \delta 144.7$ (0), 142.1 (0), 130.1 (1), 112.6 (2), 98.7 (0), 82.5 (1), 72.0 (2), 71.9 (2), 57.9 (3), 56.0 (1), 37.5 (2), 29.9 (0), 27.7 (3), 22.9 (3), 22.3 (3), 22.0 (3); $\mathrm{MS} \mathrm{m} / z 266.1876\left(\mathrm{M}^{+}\right)$, required for $\mathrm{C}_{16} \mathrm{H}_{26} \mathrm{O}_{3}: 266.1882$.

(c) To a solution of $12(329 \mathrm{mg}, 1.24 \mathrm{mmol})$ in $\mathrm{MeOH}(10 \mathrm{~mL})$ with some $\mathrm{H}_{2} \mathrm{O}(0.5 \mathrm{~mL})$ was added PPTS (32 mg, $0.13 \mathrm{mmol}$ ) at rt, and the mixture was stirred for $16 \mathrm{~h}$. Sat. $\mathrm{NaHCO}_{3}(5 \mathrm{~mL})$ was added, and the $\mathrm{MeOH}$ was evaporated under vacuum. The residue was extracted with $\mathrm{CH}_{2} \mathrm{Cl}_{2}$. The combined $\mathrm{CH}_{2} \mathrm{Cl}_{2}$ extracts were washed with brine and dried $\left(\mathrm{Na}_{2} \mathrm{SO}_{4}\right)$. The $\mathrm{CH}_{2} \mathrm{Cl}_{2}$ was removed under vacuum. Flash chromatography of the residue on a short column gave 18 (220 mg, 99\%) as an oil: ${ }^{1} \mathrm{H}$ NMR (500 $\left.\mathrm{MHz}, \mathrm{CDCl}_{3}\right): \delta 6.66(1 \mathrm{H}$, br s), $4.95(1 \mathrm{H}, \mathrm{s}), 4.85(1 \mathrm{H}, \mathrm{s}), 4.12(1 \mathrm{H}, \mathrm{m}), 3.59(1 \mathrm{H}, \mathrm{br} \mathrm{d}, J=4.0 \mathrm{~Hz})$, $3.31(3 \mathrm{H}, \mathrm{s}), 2.68-2.72(2 \mathrm{H}, \mathrm{m}), 2.35(3 \mathrm{H}, \mathrm{s}), 1.81(3 \mathrm{H}, \mathrm{s}) ;{ }^{13} \mathrm{C} \mathrm{NMR}\left(125 \mathrm{MHz}, \mathrm{CDCl}_{3}\right): \delta 196.6(0)$, 143.8 (0), 143.3 (1), 142.5 (0), 112.7 (2), 81.4 (1), 57.4 (3), 57.4 (1), 35.8 (2), 26.3 (3), 22.3 (3); MS $m / z, 180.1149\left(\mathrm{M}^{+}\right)$, required for $\mathrm{C}_{11} \mathrm{H}_{16} \mathrm{O}_{2}: 180.1150$. 


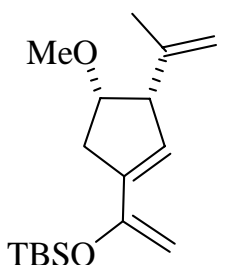

To a solution of $18(8 \mathrm{mg}, 0.04 \mathrm{mmol})$ and $\mathrm{Et}_{3} \mathrm{~N}(14 \mathrm{mg}, 0.14 \mathrm{mmol})$ in $\mathrm{CH}_{2} \mathrm{Cl}_{2}(10 \mathrm{~mL})$ was added TBS-triflate $(15 \mathrm{mg}, 0.05 \mathrm{mmol})$ at $0{ }^{\circ} \mathrm{C}$. The mixture was stirred at $0{ }^{\circ} \mathrm{C}$ for $10 \mathrm{~min}$. The solvent was removed under vacuum. The residue was passed quickly through a small column to give 19 (13 $\mathrm{mg}$, $100 \%)$ as an oil: ${ }^{1} \mathrm{H}$ NMR (500 MHz, $\left.\mathrm{CDCl}_{3}\right): \delta 5.88(1 \mathrm{H}, \mathrm{br} \mathrm{s}), 4.86(1 \mathrm{H}, \mathrm{s}), 4.81(1 \mathrm{H}, \mathrm{s}), 4.32(2 \mathrm{H}, \mathrm{s})$, $4.15(1 \mathrm{H}, \mathrm{m}), 3.51(1 \mathrm{H}, \mathrm{brd}, J=7.5 \mathrm{~Hz}), 3.34(3 \mathrm{H}, \mathrm{s}), 2.64(1 \mathrm{H}, \mathrm{dd}, J=6.9,15.7 \mathrm{~Hz}), 2.53(1 \mathrm{H}, \mathrm{dd}, J$ $=5.4,15.7 \mathrm{~Hz}), 1.78(3 \mathrm{H}, \mathrm{s}), 0.96(9 \mathrm{H}, \mathrm{s}), 0.18(6 \mathrm{H}, \mathrm{s}) ;{ }^{13} \mathrm{C} \mathrm{NMR}\left(125 \mathrm{MHz}, \mathrm{CDCl}_{3}\right): \delta 153.5(0)$, 144.6 (0), 139.3 (0), 128.7 (1), 112.2 (2), 93.5 (2), 82.4 (1), 57.7 (3), 56.0 (1), 37.4 (2), 25.8 (3), 21.9 (3), $18.2(0),-4.6(3),-4.7(3)$.

(1 $\alpha, 2 \alpha, 5 a \alpha, 9 a \alpha, 9 b \alpha)-4-(t e r t-B u t y l d i m e t h y l s i l y l o x y)-2,3,5,5 a, 9 a, 9 b$-hexahydro-1-isopropenyl-2methoxy-8,9a-dimethyl-1H-benz $[e]$ indene-6,9-dione (20)

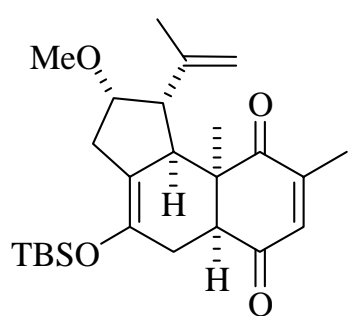

A solution of 2,6-dimethyl-para-benzoquinone (12 mg, $0.09 \mathrm{mmol})$ and 19 (13 mg, $0.04 \mathrm{mmol})$ in toluene $(10 \mathrm{~mL})$ was heated at reflux for 4 days. The solvent was removed under vacuum, and flash chromatography of the residue gave $20(15 \mathrm{mg}, 80 \%)$ as a solid: $\mathrm{mp} 95-97{ }^{\circ} \mathrm{C}$; ${ }^{1} \mathrm{H} \mathrm{NMR}(500 \mathrm{MHz}$, $\left.\mathrm{CDCl}_{3}\right): \delta 6.40(1 \mathrm{H}, \mathrm{br} \mathrm{s}), 4.85(1 \mathrm{H}, \mathrm{s}), 4.83(1 \mathrm{H}, \mathrm{s}), 3.93(1 \mathrm{H}, \mathrm{m}), 3.48(1 \mathrm{H}, \mathrm{dd}, J=6.6,8.6 \mathrm{~Hz}), 3.30$ $(3 \mathrm{H}, \mathrm{s}), 2.91(1 \mathrm{H}, \mathrm{t}, J=8.4 \mathrm{~Hz}), 2.63(2 \mathrm{H}, \mathrm{m}), 2.37(2 \mathrm{H}, \mathrm{m}), 2.15(1 \mathrm{H}, \mathrm{m}), 1.95(3 \mathrm{H}, \mathrm{s}), 1.82(3 \mathrm{H}, \mathrm{s})$, $1.36(3 \mathrm{H}, \mathrm{s}), 0.90(9 \mathrm{H}, \mathrm{s}), 0.06(6 \mathrm{H}, \mathrm{s})$; short distances identified by NOE: $9 \mathrm{a}-\mathrm{CH}_{3}$ to $5 \alpha-\mathrm{H}, 9 \mathrm{a}-\mathrm{CH}_{3}$ to 5a-H, 9a-CH $H_{3}$ to 9b-H; ${ }^{13} \mathrm{C}$ NMR (125 MHz, $\mathrm{CDCl}_{3}$ ): $\delta 202.6(0), 200.3(0), 148.5(0), 145.9(0), 138.9$ (0), 133.6 (1), 117.9 (0), 113.8 (2), 83.5 (1), 57.4 (2C, 1 and 3), 52.0 (0), 51.5 (1), 48.8 (1), 33.5 (2), 31.6 (2), 25.7 (3), 24.6 (3), 21.7 (3), 18.1 (0), 16.6 (3), -3.9 (3), -4.0 (3); MS m/z $430.2550\left(\mathrm{M}^{+}\right)$, required for $\mathrm{C}_{25} \mathrm{H}_{38} \mathrm{O}_{4} \mathrm{Si}: 430.2539$. 
$(1 \alpha, 2 \alpha, 5 a \alpha, 6 \alpha, 9 a \alpha, 9 b \alpha)-4-(t e r t-B u t y l d i m e t h y l s i l y l o x y)-2,3,5,5 a, 9 a, 9 b$-hexahydro-6-hydroxy-1-

isopropenyl-2-methoxy-8,9 $a$-dimethyl-1H-benz[e]inden-9-one $(22 a)$ and $(1 \alpha, 2 \alpha, 5 a \alpha, 6 \beta, 9 a \alpha, 9 b \alpha)$ 4-(tert-butyldimethylsilyloxy)-2,3,5,5a,9a,9b-hexahydro-6-hydroxy-1-isopropenyl-2-methoxy8,9a-dimethyl-1H-benz[e]inden-9-one (22b)

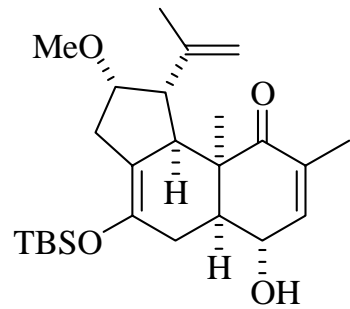

22a

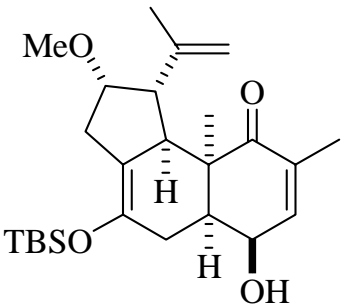

22b

A solution $\mathrm{NaBH}_{4}(1.3 \mathrm{mg}, 0.035 \mathrm{mmol})$ in $\mathrm{MeOH}(0.5 \mathrm{~mL})$ was added to a solution of 20 (15 mg, $0.035 \mathrm{mmol})$ and $\mathrm{CeCl}_{3} \cdot 7 \mathrm{H}_{2} \mathrm{O}(26 \mathrm{mg}, 0.070 \mathrm{mmol})$ in $\mathrm{MeOH}(2 \mathrm{~mL})$ at $0{ }^{\circ} \mathrm{C}$. The mixture was stirred at $0{ }^{\circ} \mathrm{C}$ for $30 \mathrm{~min}$, and it was allowed to warm to rt. Five drops of $\mathrm{H}_{2} \mathrm{O}$ were added before the mixture was concentrated under vacuum. The residue was redissolved in $\mathrm{CH}_{2} \mathrm{Cl}_{2}$, and the solution was dried over $\mathrm{Na}_{2} \mathrm{SO}_{4}$. The solvent was removed under vacuum, and flash chromatography of the residue over a very short column removed some unreacted $\mathbf{2 0}$ and provided $22(\mathbf{a} / \mathbf{b})(12 \mathrm{mg}, 80 \%)$. The isomer 22a was isolated by careful chromatography, and a chromatographic fraction enriched in $\mathbf{2 2 b}$ was obtained. For 22a: ${ }^{1} \mathrm{H}$ NMR (500 MHz, $\left.\mathrm{CDCl}_{3}\right)$ : $\delta 6.40(1 \mathrm{H}$, narrow m), $4.86(1 \mathrm{H}$, narrow m), $4.67(1 \mathrm{H}$, narrow m), $4.26(1 \mathrm{H}, \mathrm{m}), 3.82(1 \mathrm{H}, \mathrm{m}), 3.28(3 \mathrm{H}, \mathrm{s}), 2.86(1 \mathrm{H}, \mathrm{dd}, J=7.0,10.5 \mathrm{~Hz}), 2.80(1 \mathrm{H}, \mathrm{br} \mathrm{dd}, J=6.2$, $15.8 \mathrm{~Hz}), 2.57(1 \mathrm{H}, \mathrm{d}, J=10.5 \mathrm{~Hz}), 2.35(1 \mathrm{H}, \mathrm{m}), 2.08-2.18(3 \mathrm{H}, \mathrm{m}), 1.76(3 \mathrm{H}, \mathrm{br} \mathrm{s}), 1.74$ (3H, br s), $1.39(3 \mathrm{H}, \mathrm{s}), 0.94(9 \mathrm{H}, \mathrm{s}), 0.11(3 \mathrm{H}, \mathrm{s}), 0.10(3 \mathrm{H}, \mathrm{s})$; short distances identified by NOE: 1-H to 6- $\mathrm{H}$, $5 \beta-\mathrm{H}$ to $6-\mathrm{H} ;{ }^{13} \mathrm{C}$ NMR $\left(125 \mathrm{MHz}, \mathrm{CDCl}_{3}\right): \delta 202.6,144.5,140.6,138.9,135.8,118.0,114.5,83.1$, 67.8, 57.6, 53.8, 49.8, 48.1, 46.8, 33.1, 30.9, 25.7, 25.1, 21.7, 18.2, 16.4, -3.4 and -4.1 ; MS $m / z$ 432.2687 $\left(\mathrm{M}^{+}\right)$, required for $\mathrm{C}_{25} \mathrm{H}_{40} \mathrm{O}_{4} \mathrm{Si}: 432.2696$. For 22b: ${ }^{1} \mathrm{H}$ NMR $\left(500 \mathrm{MHz}, \mathrm{CDCl}_{3}\right): \delta 6.34(1 \mathrm{H}$, s), $4.94(1 \mathrm{H}$, br m), $4.86(1 \mathrm{H}, \mathrm{s}), 4.83(1 \mathrm{H}, \mathrm{s}), 3.96(1 \mathrm{H}$, apparent q, $J=6 \mathrm{~Hz}), 3.73(1 \mathrm{H}, \mathrm{t}, J=7 \mathrm{~Hz})$, $3.32(3 \mathrm{H}, \mathrm{s}), 2.53-2.65(3 \mathrm{H}, \mathrm{m}), 2.37(1 \mathrm{H}, \mathrm{m}), 2.25(1 \mathrm{H}, \mathrm{m}), 2.01(1 \mathrm{H}, \mathrm{m}), 1.85(3 \mathrm{H}, \mathrm{s}), 1.76(3 \mathrm{H}, \mathrm{s})$, $0.94(3 \mathrm{H}, \mathrm{s}), 0.91$ (9H, s), 0.04 (6H, s). 
$\left(1 R^{*}, 2 S^{*}, 3 S^{*}, 4 R^{*}, 5 S^{*}, 7 R^{*}, 8 R^{*}, 10 R^{*}\right)$-1-Allyl-8-(tert-butyldimethylsilyloxy)-4-isopropenyl-5methoxy-2,13-dimethyl-14-oxatetracyclo[6.5.1.0 $\left.0^{2,10} .0^{3,7}\right]$ tetradec-12-en-11-one (23)

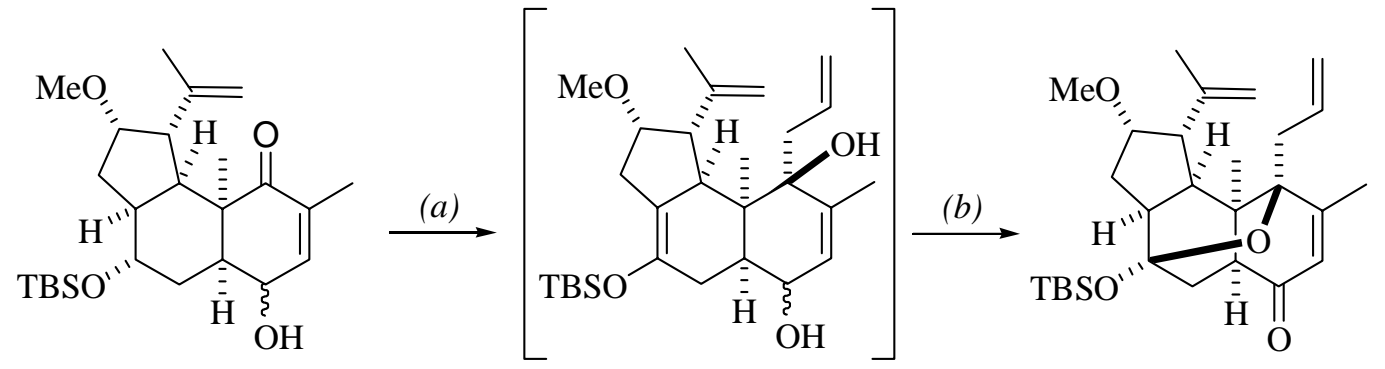

(a) Allyl bromide (163 mg, $1.35 \mathrm{mmol}$ ) was added dropwise to magnesium turnings (37 mg, 1.50 mmol) in dry ether $(10 \mathrm{~mL})$. This was stirred for $30 \mathrm{~min}$ at $\mathrm{rt}$, as the solution became cloudy, and then at reflux for a further $30 \mathrm{~min}$. A portion of the solution of allylmagnesium bromide $(2.0 \mathrm{~mL}, 0.27$ mmol) was added to a solution of $22(39 \mathrm{mg}, 0.090 \mathrm{mmol})$ in dry ether $(3.0 \mathrm{~mL})$ at $0{ }^{\circ} \mathrm{C}$. The mixture was stirred at $0{ }^{\circ} \mathrm{C}$ for $1 \mathrm{~h}$ before it was allowed to warm to $\mathrm{rt} . \mathrm{H}_{2} \mathrm{O}$ was added, and this was extracted with ether. The organic solution was dried over $\mathrm{Na}_{2} \mathrm{SO}_{4}$ and concentrated under vacuum. The residue was used directly in the next step. (The product and 22a had the same $\mathrm{R}_{f}$ on TLC, run with EtOAchexanes.)

(b) A solution of the product (containing some 22a) of (a) and Dess-Martin periodinane (76 mg, 0.18 mmol) in $\mathrm{CH}_{2} \mathrm{Cl}_{2}(5 \mathrm{~mL})$, to which $\mathrm{NaHCO}_{3}(0.8 \mathrm{~g})$ was added, was stirred at rt for $14 \mathrm{~h}$. $\mathrm{More} \mathrm{CH}_{2} \mathrm{Cl}_{2}$ was added, and the mixture was washed with $10 \% \mathrm{Na}_{2} \mathrm{~S}_{2} \mathrm{O}_{3}$, saturated $\mathrm{NaHCO}_{3}$, and brine. The organic layer was dried over $\mathrm{Na}_{2} \mathrm{SO}_{4}$, and the solvent was removed under vacuum. TLC analysis showed just two components in the residue, 23 and 20. Compound 23 (12 $\mathrm{mg}, 28 \%$ ) was obtained by flash chromatography as an oil: ${ }^{1} \mathrm{H}$ NMR $\left(500 \mathrm{MHz} \mathrm{CDCl}_{3}\right): \delta 5.79(1 \mathrm{H}, \mathrm{s}), 5.71(1 \mathrm{H}, \mathrm{m}), 5.12(1 \mathrm{H}, \mathrm{d}, J=$ $17.3 \mathrm{~Hz}), 5.07(1 \mathrm{H}, \mathrm{d}, J=10.2 \mathrm{~Hz}), 4.92(1 \mathrm{H}, \mathrm{s}), 4.84(1 \mathrm{H}, \mathrm{s}), 3.87(1 \mathrm{H}, \mathrm{br} \mathrm{t}, J=3.8 \mathrm{~Hz}), 3.28(3 \mathrm{H}, \mathrm{s})$, $3.12(1 \mathrm{H}, \mathrm{dd}, J=4.5,9.5 \mathrm{~Hz}), 2.88(1 \mathrm{H}, \mathrm{d}$ of multiplets, $J=16.9 \mathrm{~Hz}), 2.77(1 \mathrm{H}, \mathrm{dd}, J=8.8,16.9 \mathrm{~Hz})$, $2.50(1 \mathrm{H}, \mathrm{m}), 2.42(1 \mathrm{H}, \mathrm{dd}, J=9.5,12.0 \mathrm{~Hz}), 2.29(1 \mathrm{H}, \mathrm{dd}, J=4.8,11.0 \mathrm{~Hz}), 2.04(3 \mathrm{H}, \mathrm{s}), 2.01-2.06$ $(2 \mathrm{H}, \mathrm{m}), 1.86(1 \mathrm{H}, \mathrm{m}), 1.84(3 \mathrm{H}, \mathrm{s}), 1.80(1 \mathrm{H}, \mathrm{dd}, J=4.8,13.8 \mathrm{~Hz}), 0.93(3 \mathrm{H}, \mathrm{s}), 0.86(9 \mathrm{H}, \mathrm{s}), 0.12$ $(3 \mathrm{H}, \mathrm{s}), 0.05(3 \mathrm{H}, \mathrm{s})$; short distances identified by NOE and NOESY spectra: 2- $\mathrm{CH}_{3}$ to $4-\mathrm{H}, 2-\mathrm{CH}_{3}$ to 10-H, 2- $\mathrm{CH}_{3}$ to $\mathrm{CH}_{2}-\mathrm{CH}=\mathrm{CH}_{2}, 2-\mathrm{CH}_{3}$ to $\mathrm{CH}_{2}-\mathrm{CH}=\mathrm{CH}_{2}, 3-\mathrm{H}$ to $10-\mathrm{H}, 4-\mathrm{H}$ to $5-\mathrm{H}, 4-\mathrm{H}$ to $\mathrm{CH}_{2}-\mathrm{CH}^{-} \mathrm{CH}_{2}$ ( $\delta$ 2.77), 6- $\alpha \mathrm{H}$ to $7-\mathrm{H}, 13-\mathrm{CH}_{3}$ to $\mathrm{CH}_{2}-\mathrm{CH}=\mathrm{CH}_{2}, 13-\mathrm{CH}_{3}$ to $\mathrm{CH}_{2}-\mathrm{CH}=\mathrm{CH}_{2} ;{ }^{13} \mathrm{C}$ NMR (125 MHz, $\mathrm{CDCl}_{3}$ ): $\delta 201.9(0), 160.3$ (0), 144.7 (0), 134.0 (1), 124.3 (1), 116.7 (2), 112.0 (2), 99.2 (0), 85.1 (1), 80.2 (0), 56.9 (3), 56.0 (1), 54.7 (1), 49.0 (1), 46.4 (1), 40.5 (2), 39.3 (0), 35.9 (2), 30.5 (2), 25.7 (3C, 3), 23.0 (3), 20.0 (3), 19.4 (3), 17.9 (3), -2.3 (3), -3.0 (3). 
$\left(1 R^{*}, 5 R^{*}, 7 R^{*}, 8 R^{*}, 10 R^{*}, 11 R^{*}, 15 S^{*}, 16 S^{*}\right)-7-($ tert-Butyldimethylsilyloxy)-10-methoxy-3,12,15trimethyl-17-oxapentacyclo $\left[6.6 .2 .1^{1,7} \cdot 0^{5,15} \cdot 0^{11,16}\right]$ heptadeca-2,12-dien-4-one (24)

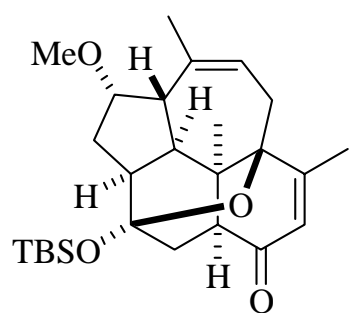

A mixture of $23(10 \mathrm{mg}, 0.021 \mathrm{mmol})$ in $\mathrm{C}_{6} \mathrm{D}_{6}(5.0 \mathrm{~mL})$ and $25\left(0.5 \mathrm{mg}, 6 \times 10^{-4} \mathrm{mmol}\right)$ was heated at reflux for $1 \mathrm{~h}$. The solution was concentrated under vacuum. Compound 24 (5 mg, 52\%) was isolated by flash chromatography as an oil: IR (neat): $1688 \mathrm{~cm}^{-1} ;{ }^{1} \mathrm{H}$ NMR $\left(500 \mathrm{MHz}, \mathrm{CDCl}_{3}\right): \delta 5.57(1 \mathrm{H}$, narrow m), $5.15(1 \mathrm{H}$, br d, $J=8.0 \mathrm{~Hz}), 3.73(1 \mathrm{H}$, apparent t, $J=3.5 \mathrm{~Hz}), 3.38(1 \mathrm{H}, \mathrm{br} \mathrm{d}, J=13.2 \mathrm{~Hz})$, $2.69(1 \mathrm{H}, \mathrm{br} \mathrm{d}, J=17.8 \mathrm{~Hz}), 2.41(1 \mathrm{H}, \mathrm{dd}, J=6.1,10.5 \mathrm{~Hz}), 2.26(1 \mathrm{H}, \mathrm{dd}, J=8.0,17.8 \mathrm{~Hz}), 2.25(1 \mathrm{H}$, $\mathrm{dd}, J=8.8,13.2 \mathrm{~Hz}), 2.11(1 \mathrm{H}, \mathrm{td}, J=8.8,4.3 \mathrm{~Hz}), 2.01(3 \mathrm{H}, \mathrm{d}, J=1.5 \mathrm{~Hz}), 1.96(1 \mathrm{H}, \mathrm{dd}, J=6.1$, $13.5 \mathrm{~Hz}), 1.90(1 \mathrm{H}, \mathrm{dd}, J=4.3,14.8 \mathrm{~Hz}), 1.84(3 \mathrm{H}, \mathrm{br}$ s), $1.84(1 \mathrm{H}$, overlapped $), 1.76(1 \mathrm{H}, \mathrm{dd}, J=8.8$, $14.8 \mathrm{~Hz}), 1.06(3 \mathrm{H}, \mathrm{s}), 0.86(9 \mathrm{H}, \mathrm{s}), 0.14(3 \mathrm{H}, \mathrm{s}), 0.07(3 \mathrm{H}, \mathrm{s}) ;{ }^{13} \mathrm{C} \mathrm{NMR}\left(125 \mathrm{MHz}, \mathrm{CDCl}_{3}\right): \delta 202.3$ (0), 159.4 (0), 139.3 (0), 121.8 (1), 102.0 (0), 80.7 (1), 79.5 (0), 56.1 (3), 54.2 (1), 49.9 (1), 46.7 (1), 43.4 (1), 39.6 (2), 39.0 (2), 37.3 (0), 29.7 (2), 25.7 (3), 20.0 (3), 18.6 (3), 18.0 (3), 18.0 (0), -2.2 (3), 2.9 (3); MS m/z $444.2692\left(\mathrm{M}^{+}\right)$, required for $\mathrm{C}_{26} \mathrm{H}_{40} \mathrm{O}_{4} \mathrm{Si}$ : 444.2696. 
NMR spectra of $\left(1 R^{*}, 2 S^{*}\right)$-2-(2-hydroxyethyl)-4-(2,5,5-trimethyl-[1,3]dioxan-2-yl)cyclopent-3-en-1-ol (14)

${ }^{1} \mathrm{H}$ NMR (500 MHz) in $\mathrm{CDCl}_{3}$<smiles>CC1CC(C2(C)OCC(C)(C)CO2)=C[C@H](CCO)C1</smiles>
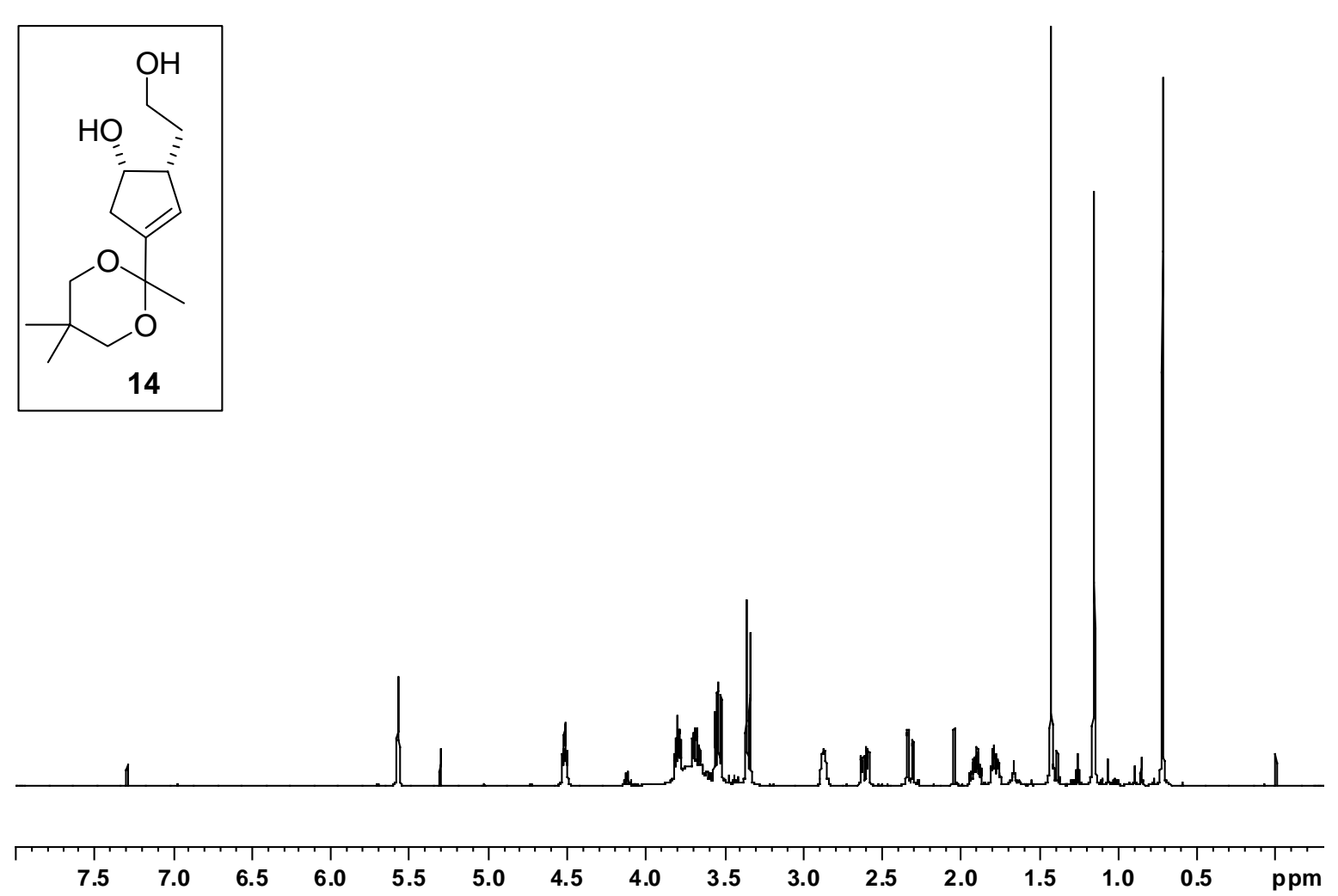

${ }^{13} \mathrm{C}$ NMR (125 MHz) in $\mathrm{CDCl}_{3}$

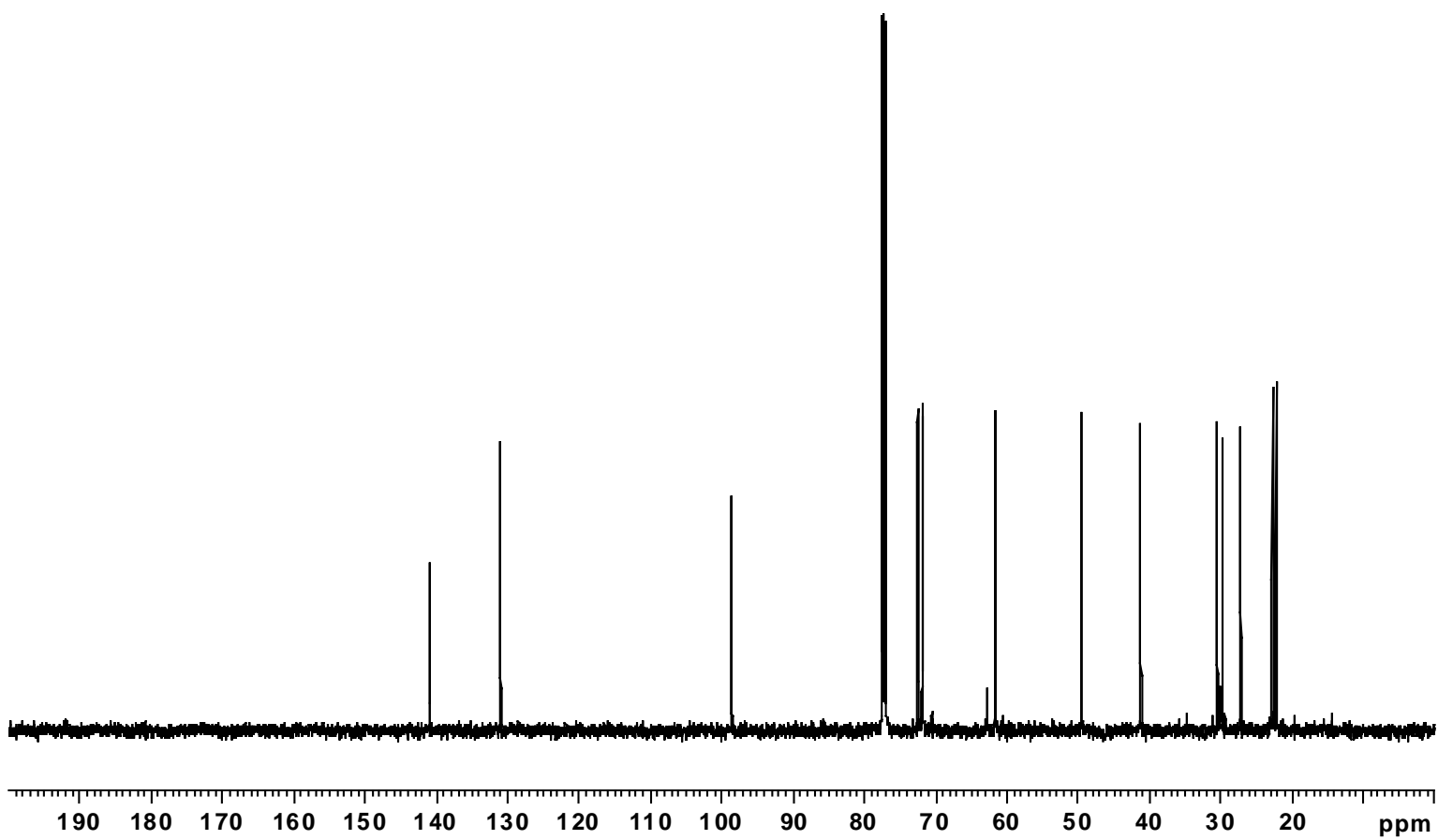


NMR spectra of $\left(3 R^{*}, 4 S^{*}\right)$-3-(2-hydroxyethyl)-4-methoxy-1-(2,5,5-trimethyl-[1,3]dioxan-2yl)cyclopentene (15)

${ }^{1} \mathrm{H}$ NMR $(500 \mathrm{MHz})$ in $\mathrm{CDCl}_{3}$

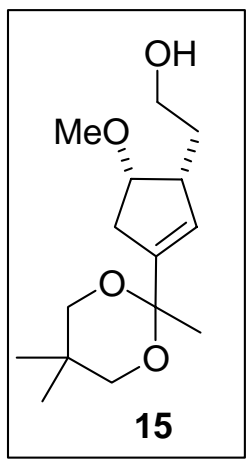

${ }^{13} \mathrm{C}$ NMR $(125 \mathrm{MHz})$ in $\mathrm{CDCl}_{3}$

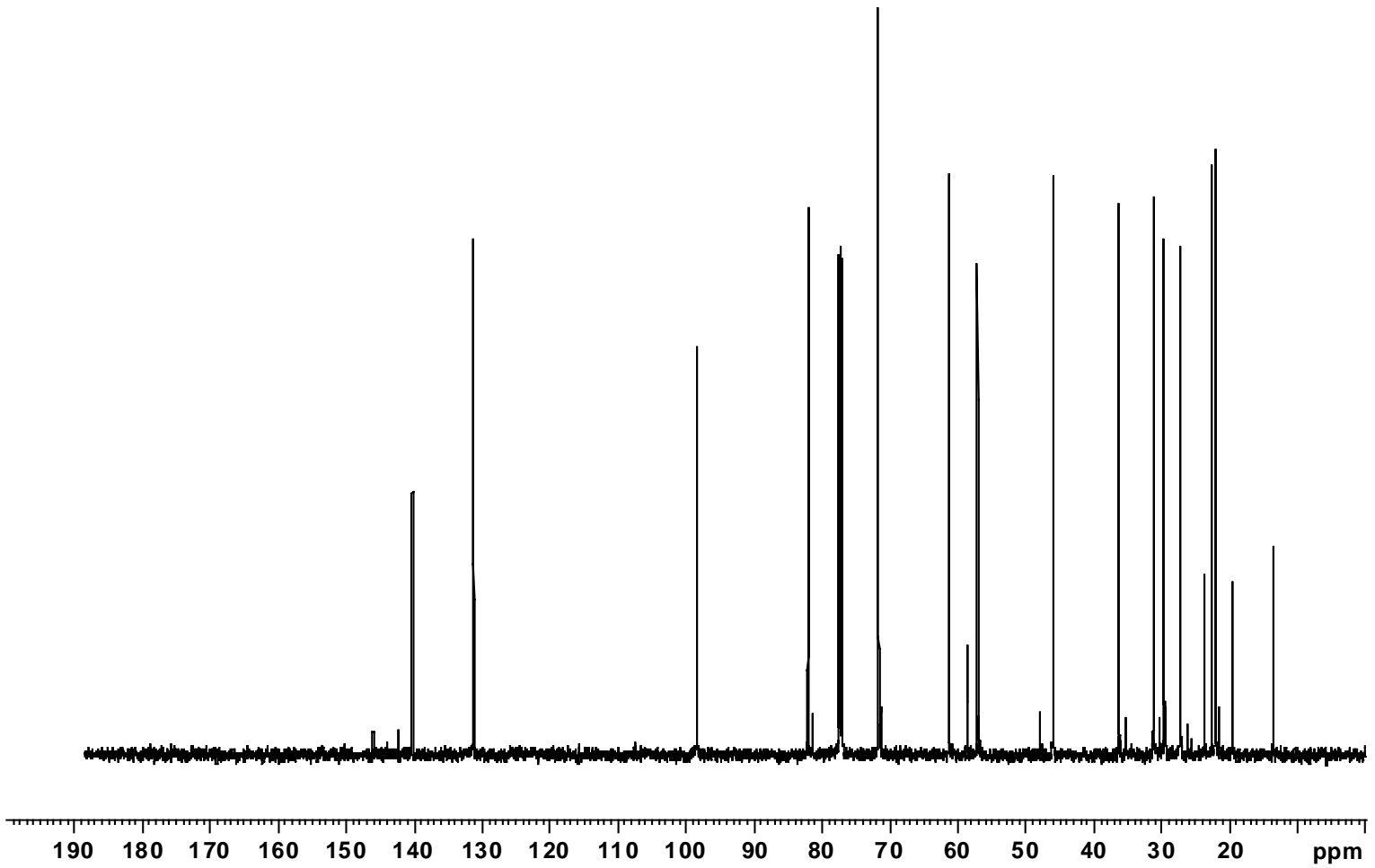


NMR spectra of $\left(3 R^{*}, 4 R^{*}\right)$-4-methoxy-3-(1-oxo-2-propen-2-yl)-1-(2,5,5-trimethyl-[1,3]dioxan-2-yl)cyclopentene (16)

${ }^{1} \mathrm{H}$ NMR $(500 \mathrm{MHz})$ in $\mathrm{CDCl}_{3}$
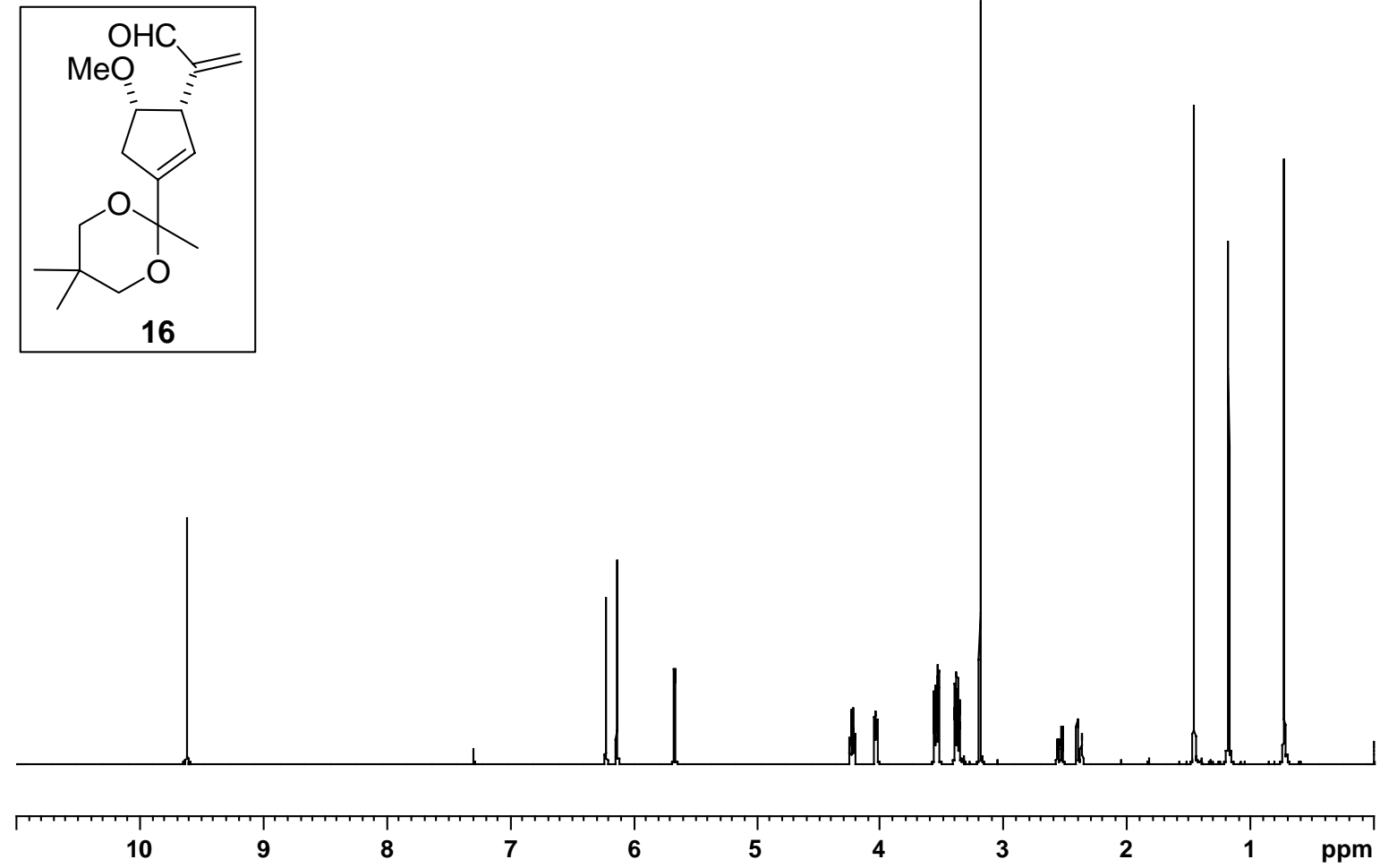

${ }^{13} \mathrm{C}$ NMR (125 MHz) in $\mathrm{CDCl}_{3}$

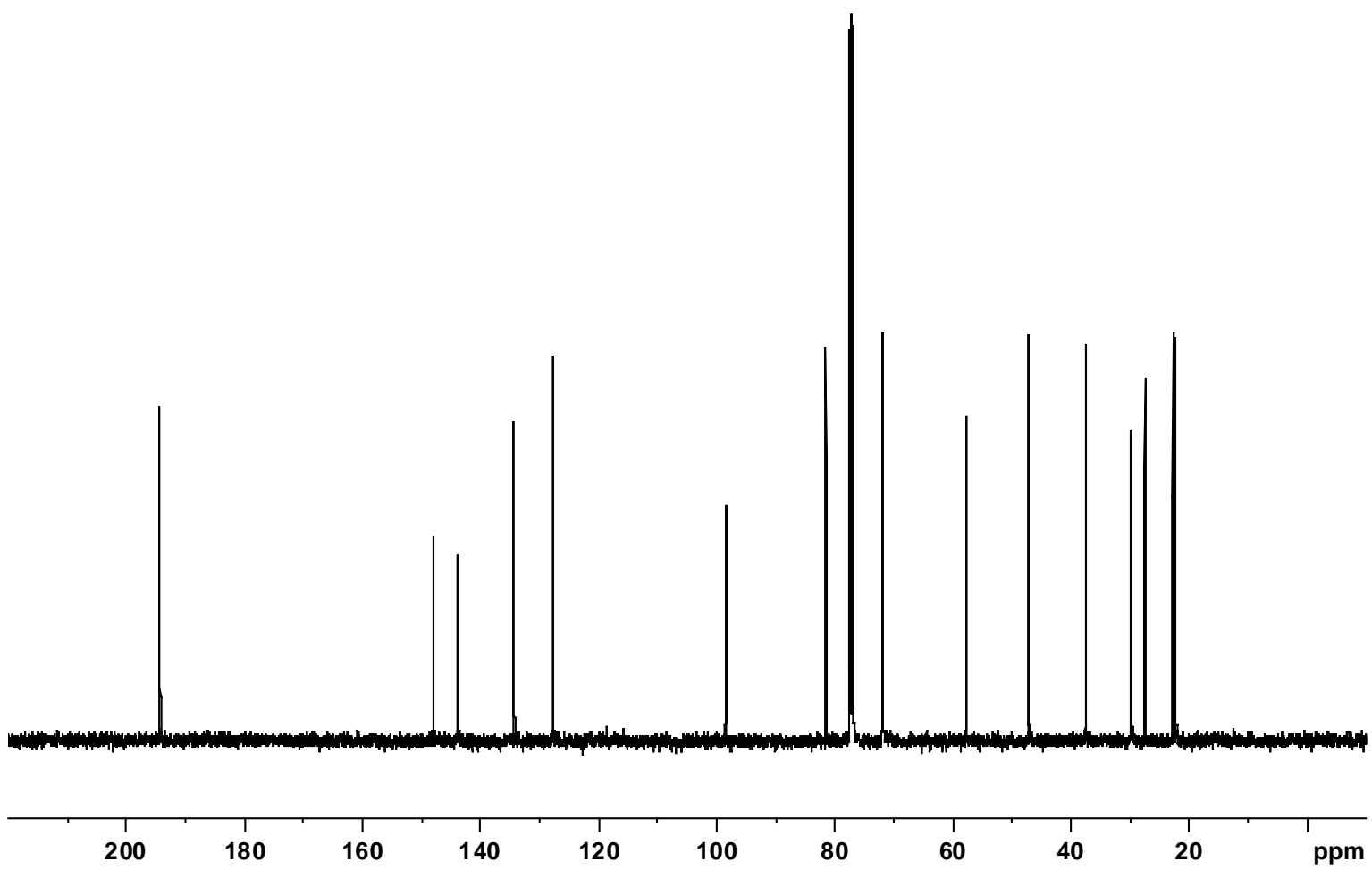


NMR spectra of $\left(3 R^{*}, 4 R^{*}\right)-3$-[1-(hydroxymethyl)vinyl]-4-methoxy-1-(2,5,5-trimethyl-[1,3]dioxan-2-yl)cyclopentene (17)

${ }^{1} \mathrm{H} \mathrm{NMR}(500 \mathrm{MHz})$ in $\mathrm{CDCl}_{3}$<smiles>C=C(CO)[C@H]1C=C(C2(C)OCC(C)(C)CO2)C[C@H]1OC</smiles>
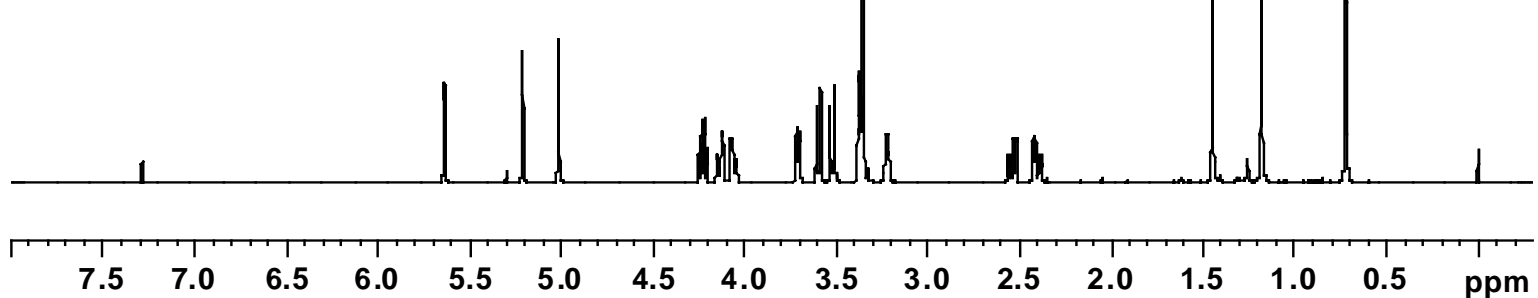

${ }^{13} \mathrm{C}$ NMR $(125 \mathrm{MHz})$ in $\mathrm{CDCl}_{3}$

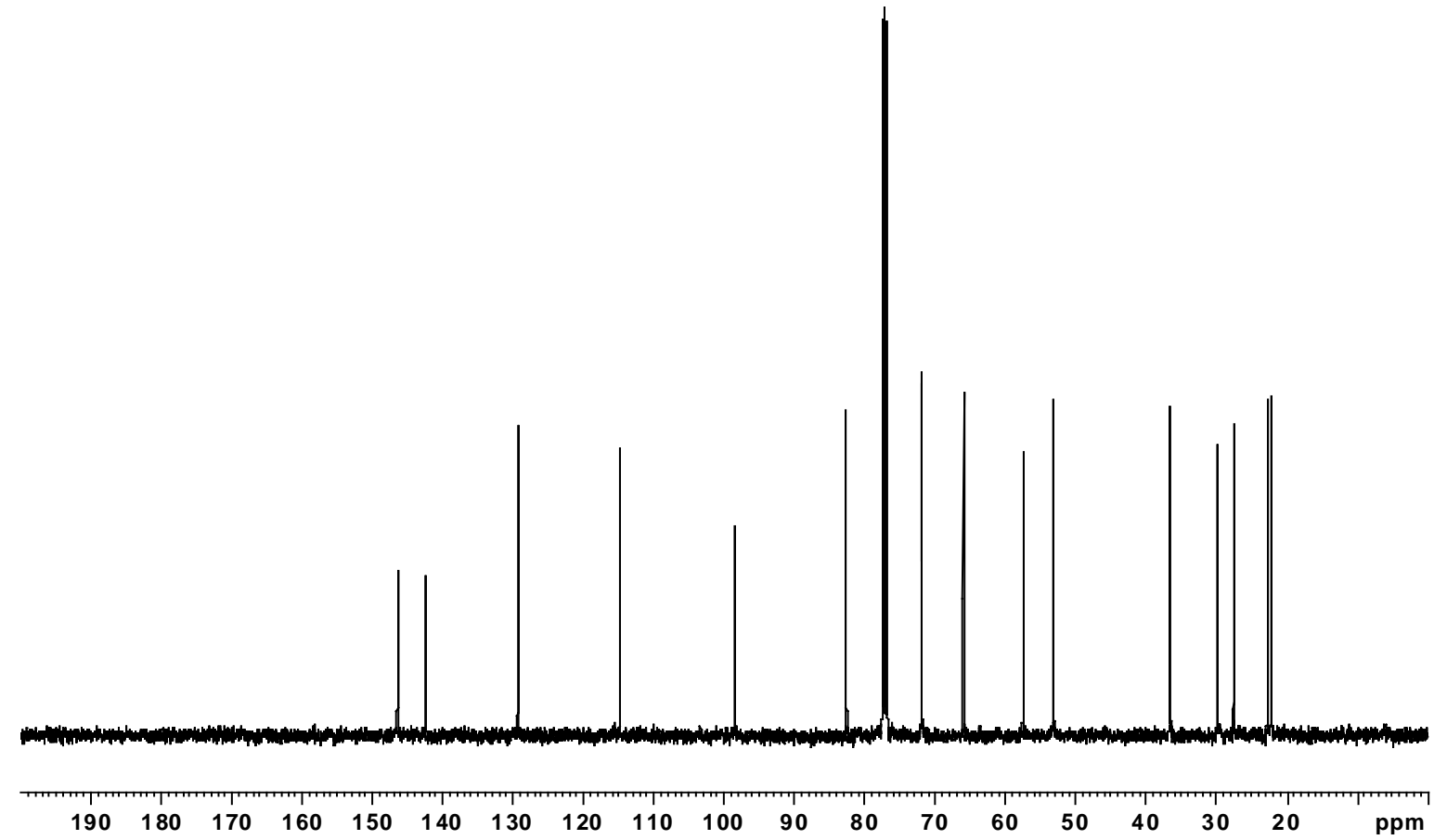


NMR spectra of $\left(3 R^{*}, 4 R^{*}\right)$-1-acetyl-3-isopropenyl-4-methoxycyclopentene (18)

${ }^{1} \mathrm{H} \mathrm{NMR}(500 \mathrm{MHz})$ in $\mathrm{CDCl}_{3}$
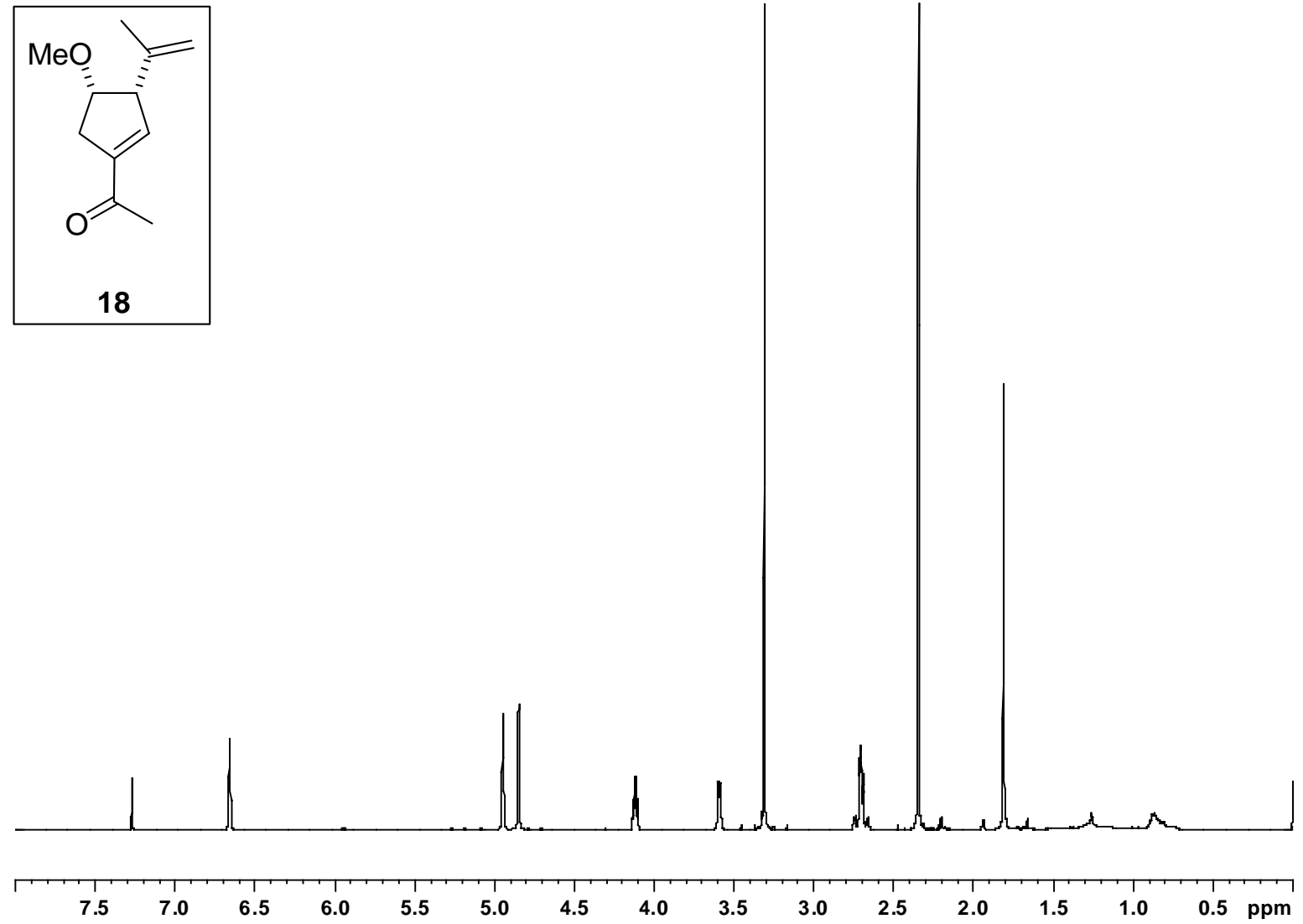

${ }^{13} \mathrm{C} \mathrm{NMR}(125 \mathrm{MHz})$ in $\mathrm{CDCl}_{3}$

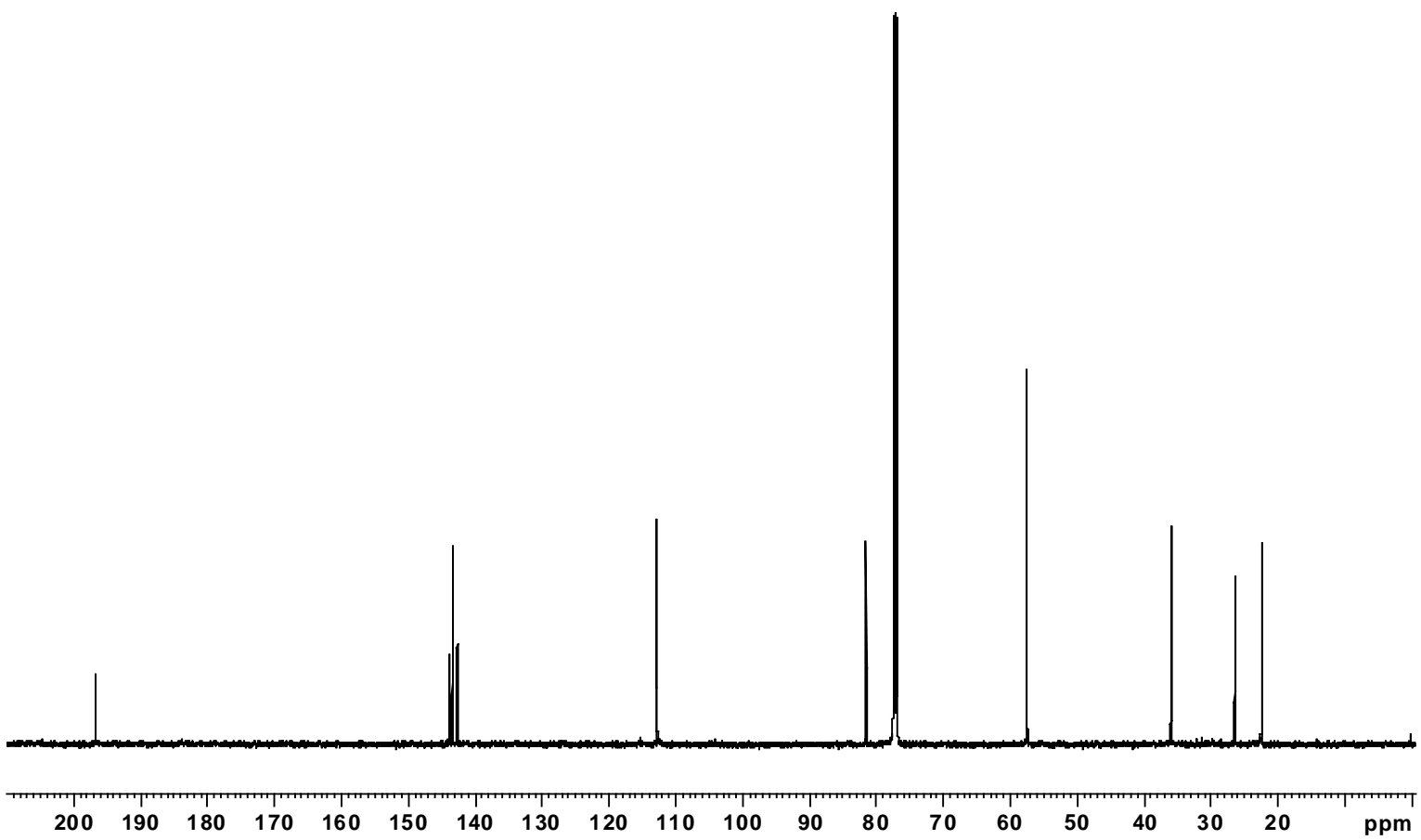


NMR spectra of $\left(3 R^{*}, 4 R^{*}\right)$-1-(1-tert-butyldimethylsilyloxy vinyl)-3-isopropenyl-4-methoxycyclopentene (19)

${ }^{1} \mathrm{H}$ NMR $(500 \mathrm{MHz})$ in $\mathrm{CDCl}_{3}$
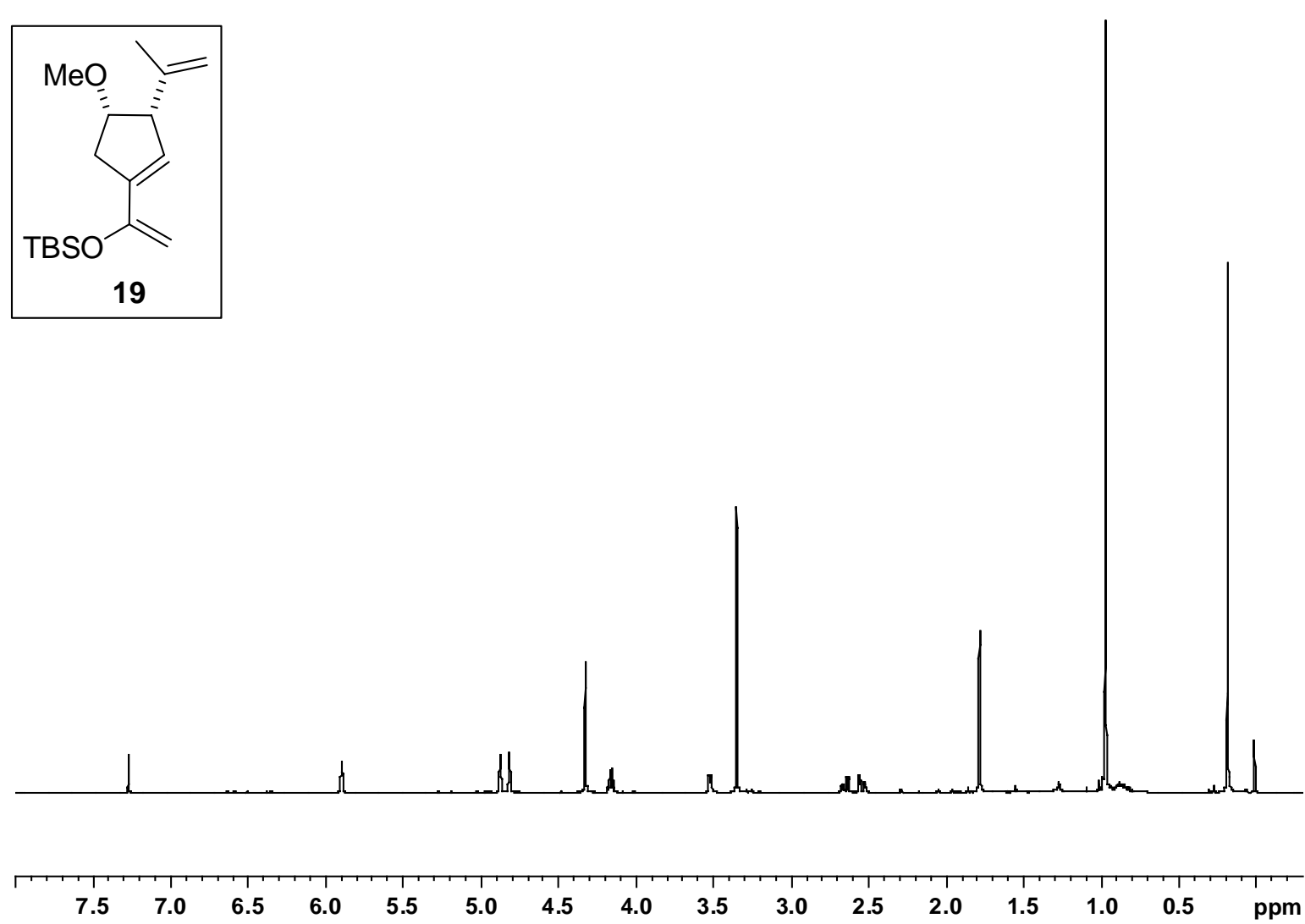

${ }^{13} \mathrm{C}$ NMR $(125 \mathrm{MHz})$ in $\mathrm{CDCl}_{3}$

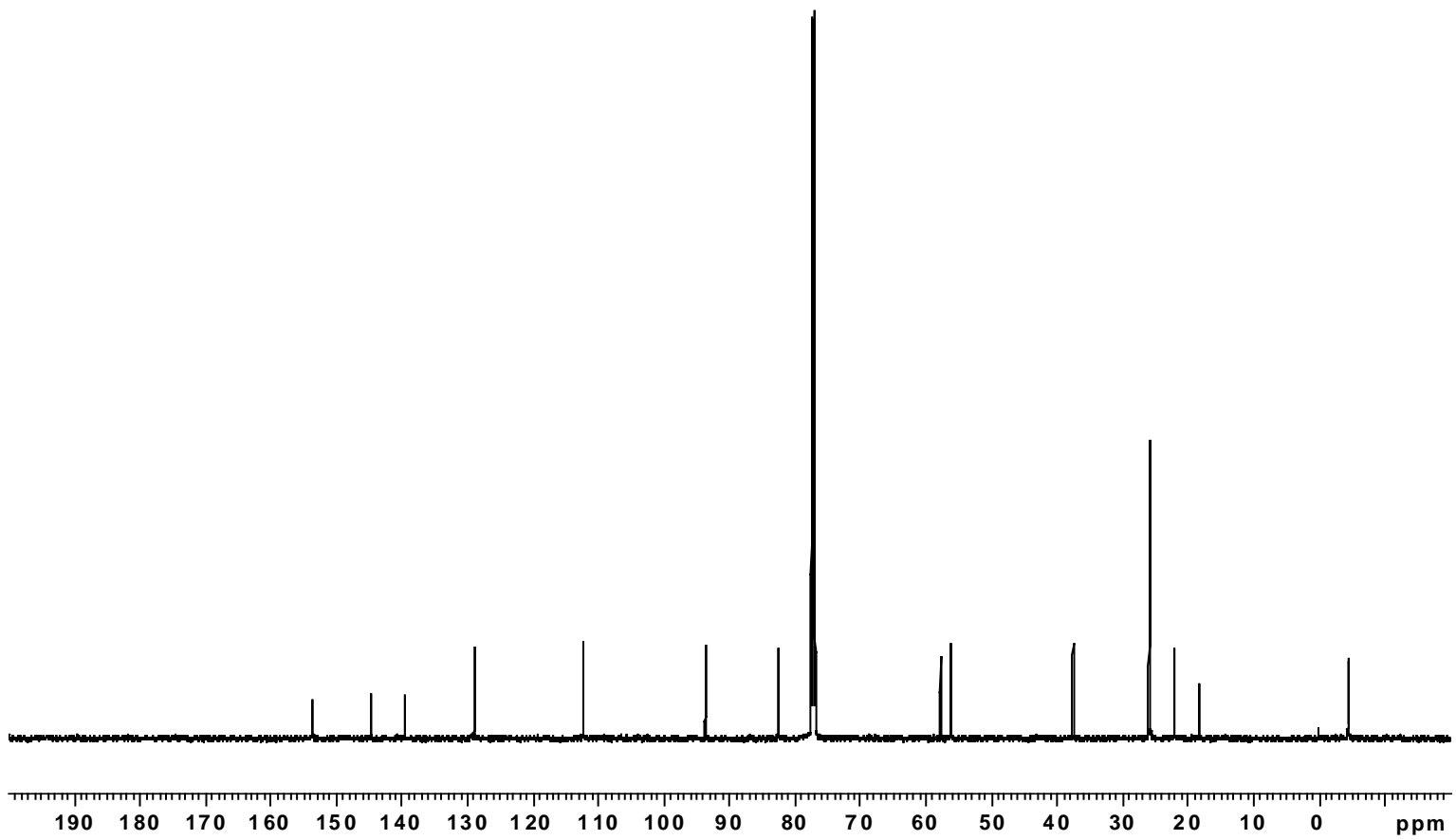


NMR spectra of $(1 \alpha, 2 \alpha, 5 a \alpha, 9 a \alpha, 9 b \alpha)$-4-(tert-butyldimethylsilyloxy)-2,3,5,5a,9a,9b-hexahydro-1-isopropenyl-2-methoxy-8,9a-dimethyl-1H-benz $[e]$ indene-6,9-dione (20)

${ }^{1} \mathrm{H}$ NMR $(500 \mathrm{MHz})$ in $\mathrm{CDCl}_{3}$
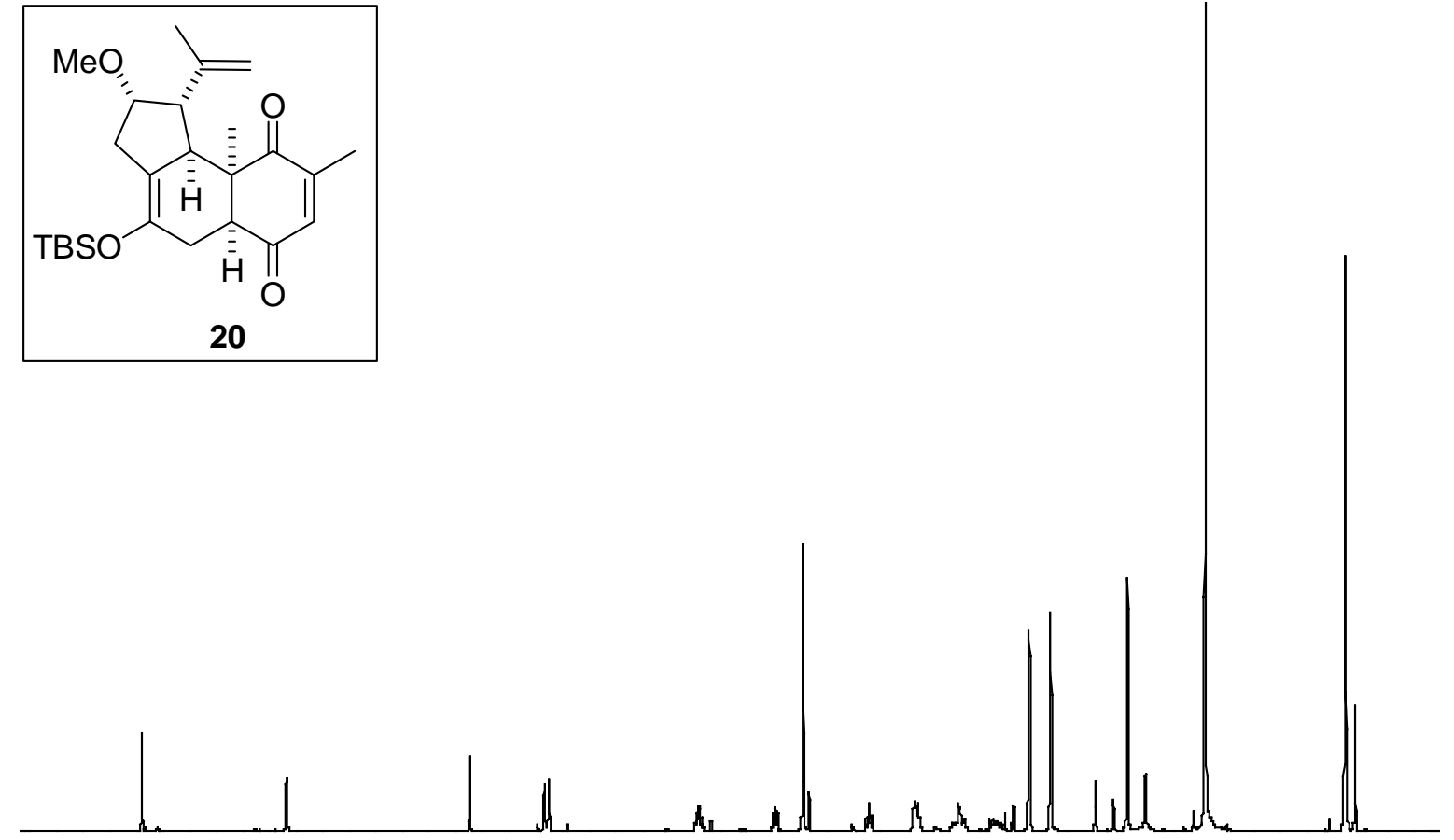

\begin{tabular}{|c|c|c|c|c|c|c|c|c|c|c|c|c|c|c|c|}
\hline$T$ & $T$ & $T$ & $T$ & $T$ & $T$ & 7 & $T$ & $T$ & $T$ & $T$ & $T$ & $T$ & $T$ & $T$ & \\
\hline 7.5 & 7.0 & 6.5 & 6.0 & 5.5 & 5.0 & 4.5 & 4.0 & 3.5 & 3.0 & 2.5 & 2.0 & 1.5 & 1.0 & 0.5 & ppm \\
\hline
\end{tabular}

${ }^{13} \mathrm{C}$ NMR (125 MHz) in $\mathrm{CDCl}_{3}$

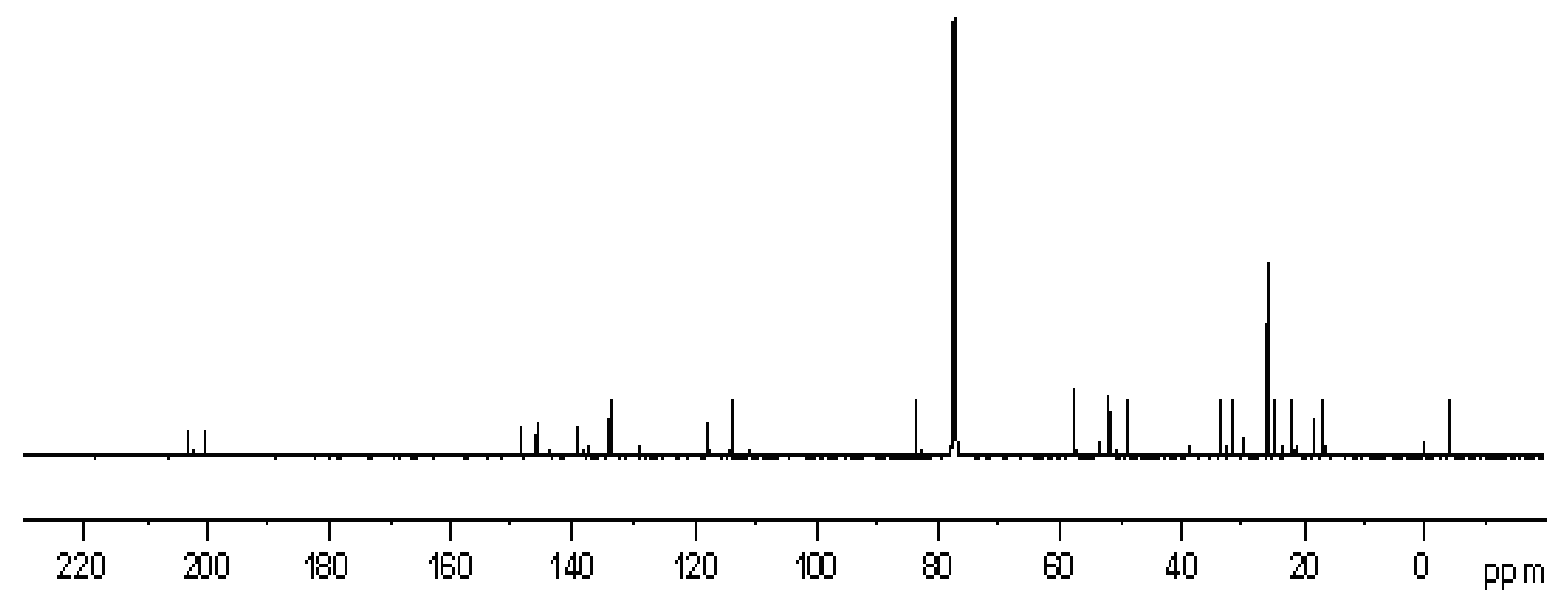


NMR spectra of $(1 \alpha, 2 \alpha, 5 a \alpha, 6 \alpha, 9 a \alpha, 9 b \alpha)-4$-(tert-butyldimethylsilyloxy)-2,3,5,5a,9a,9bhexa-hydro-6-hydroxy-1-isopropenyl-2-methoxy-8,9a-dimethyl-1H-benz $[e]$ inden-9-one (22a)

${ }^{1} \mathrm{H}$ NMR $(500 \mathrm{MHz})$ in $\mathrm{CDCl}_{3}$

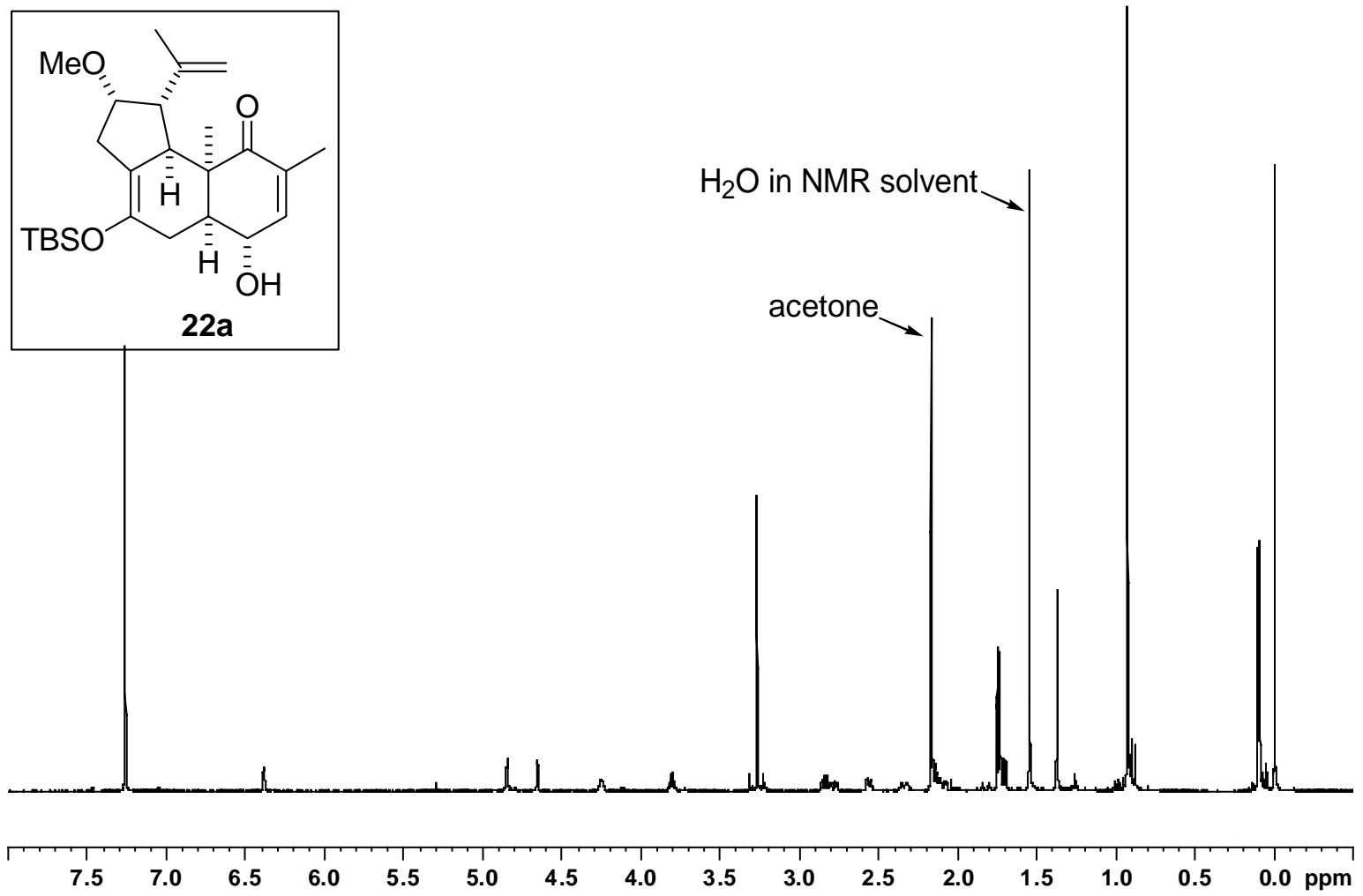

${ }^{13} \mathrm{C}$ NMR $(125 \mathrm{MHz})$ in $\mathrm{CDCl}_{3}$

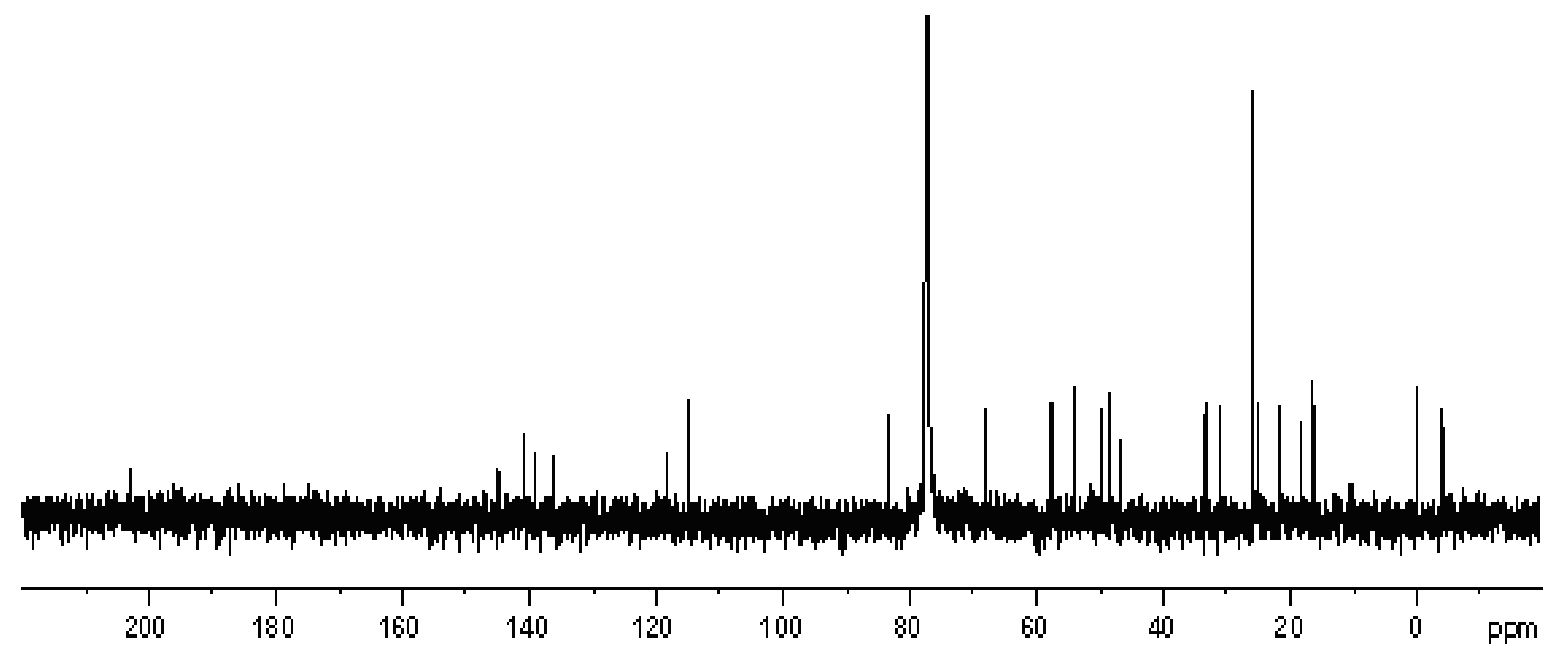


NMR spectra of $\left(1 R^{*}, 2 S^{*}, 3 S^{*}, 4 R^{*}, 5 S^{*}, 7 R^{*}, 8 R^{*}, 10 R^{*}\right)$-1-allyl-8-(tert-butyldimethylsilyloxy)-4-isopropenyl-5-methoxy-2,13-dimethyl-14-oxatetracyclo $\left[6.5 .1 .0^{2,10} .^{3,7}\right]$ tetradec-12en-11-one (23)

${ }^{1} \mathrm{H}$ NMR $(500 \mathrm{MHz})$ in $\mathrm{CDCl}_{3}$
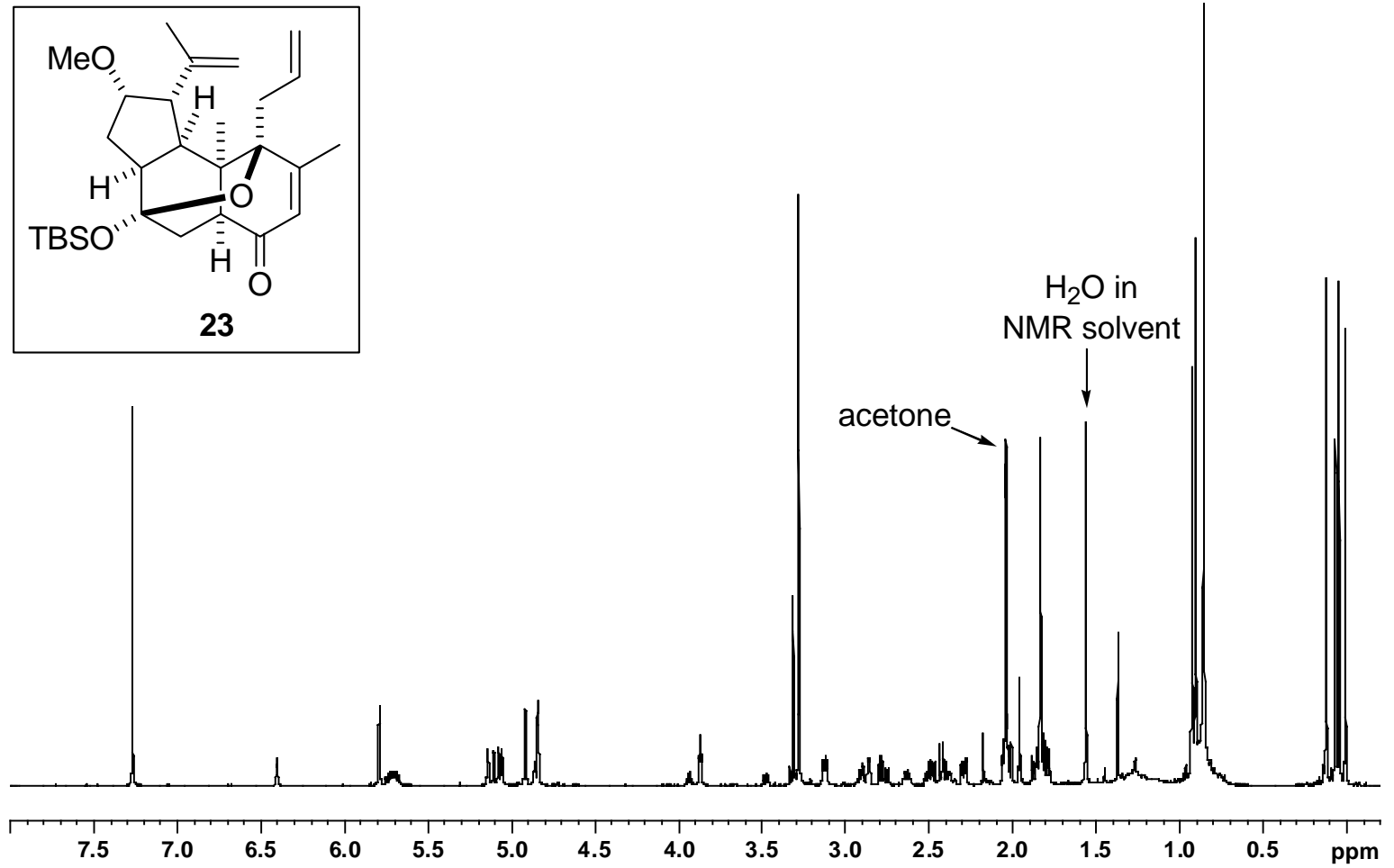

${ }^{13} \mathrm{C} \mathrm{NMR}(125 \mathrm{MHz})$ in $\mathrm{CDCl}_{3}$

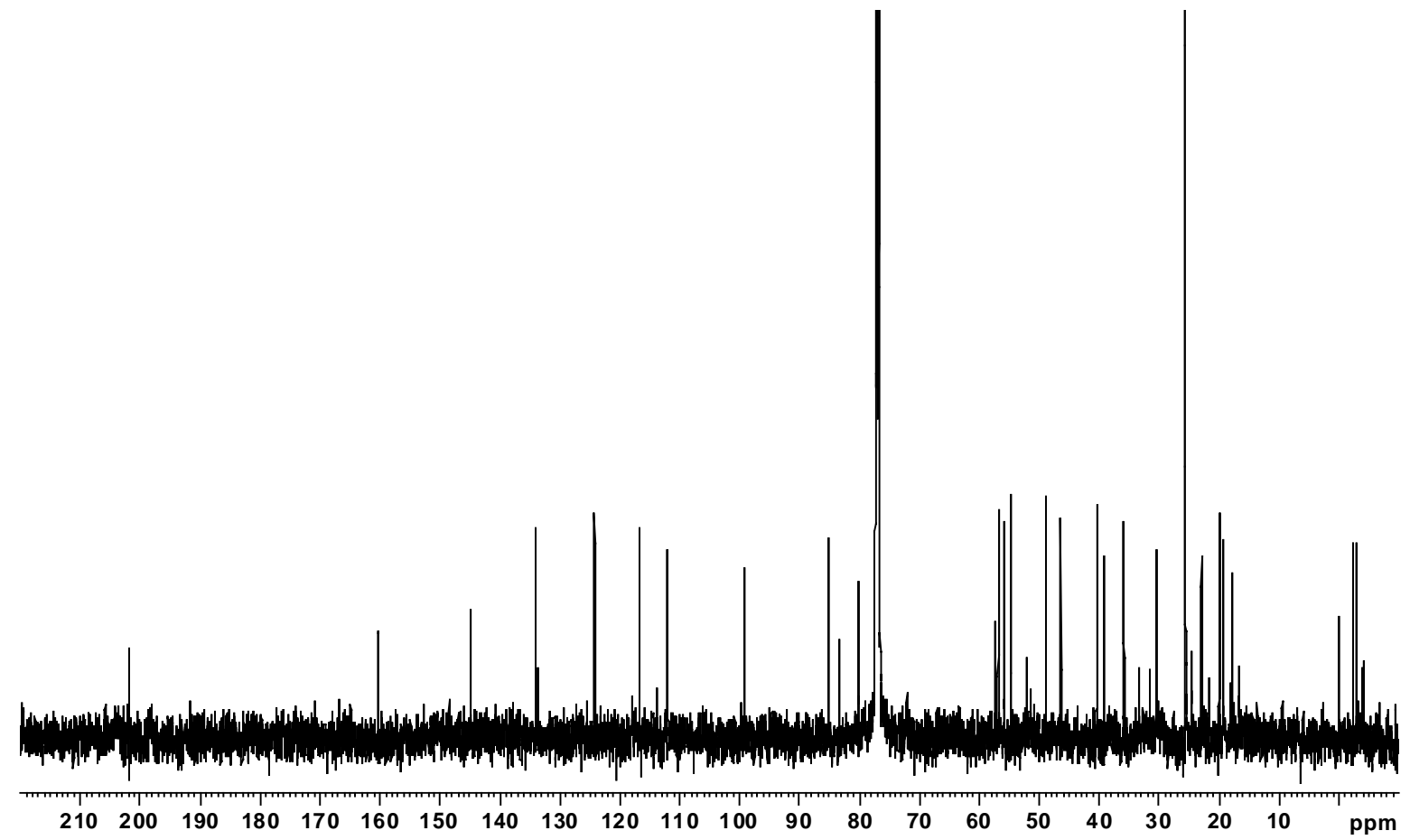


NMR spectra of $\left(1 R^{*}, 5 R^{*}, 7 R^{*}, 8 R^{*}, 10 R^{*}, 11 R^{*}, 15 S^{*}, 16 S^{*}\right)$-7-(tert-butyldimethylsilyloxy)10-methoxy-3,12,15-trimethyl-17-oxapentacyclo[6.6.2.1 $\left.{ }^{1,7} \cdot 0^{5,15} \cdot 0^{11,16}\right]$ heptadeca-2,12-dien-4one (24)

${ }^{1} \mathrm{H}$ NMR $(500 \mathrm{MHz})$ in $\mathrm{CDCl}_{3}$
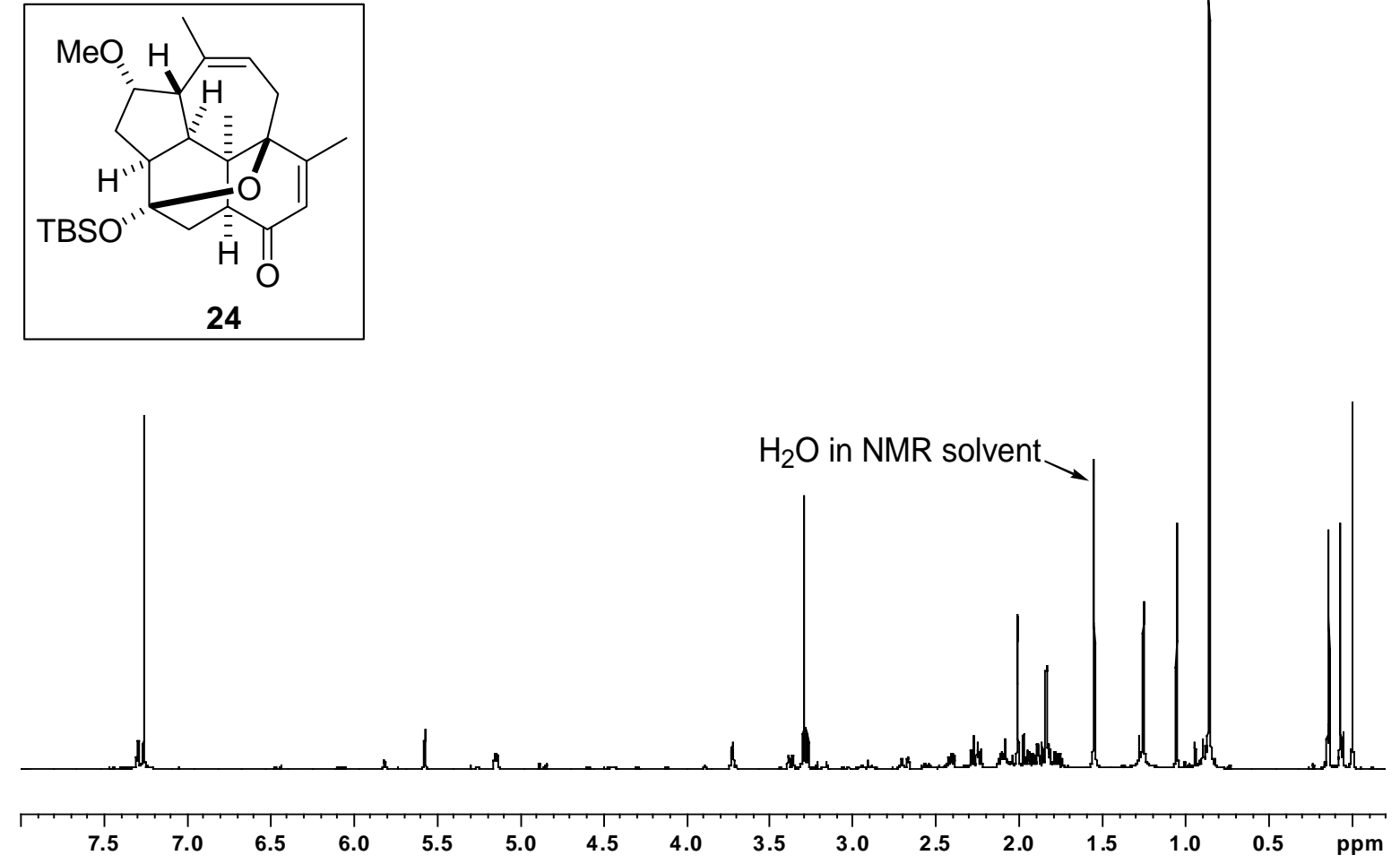

JMOD ${ }^{13} \mathrm{C}$ NMR (125 MHz) in $\mathrm{CDCl}_{3}$

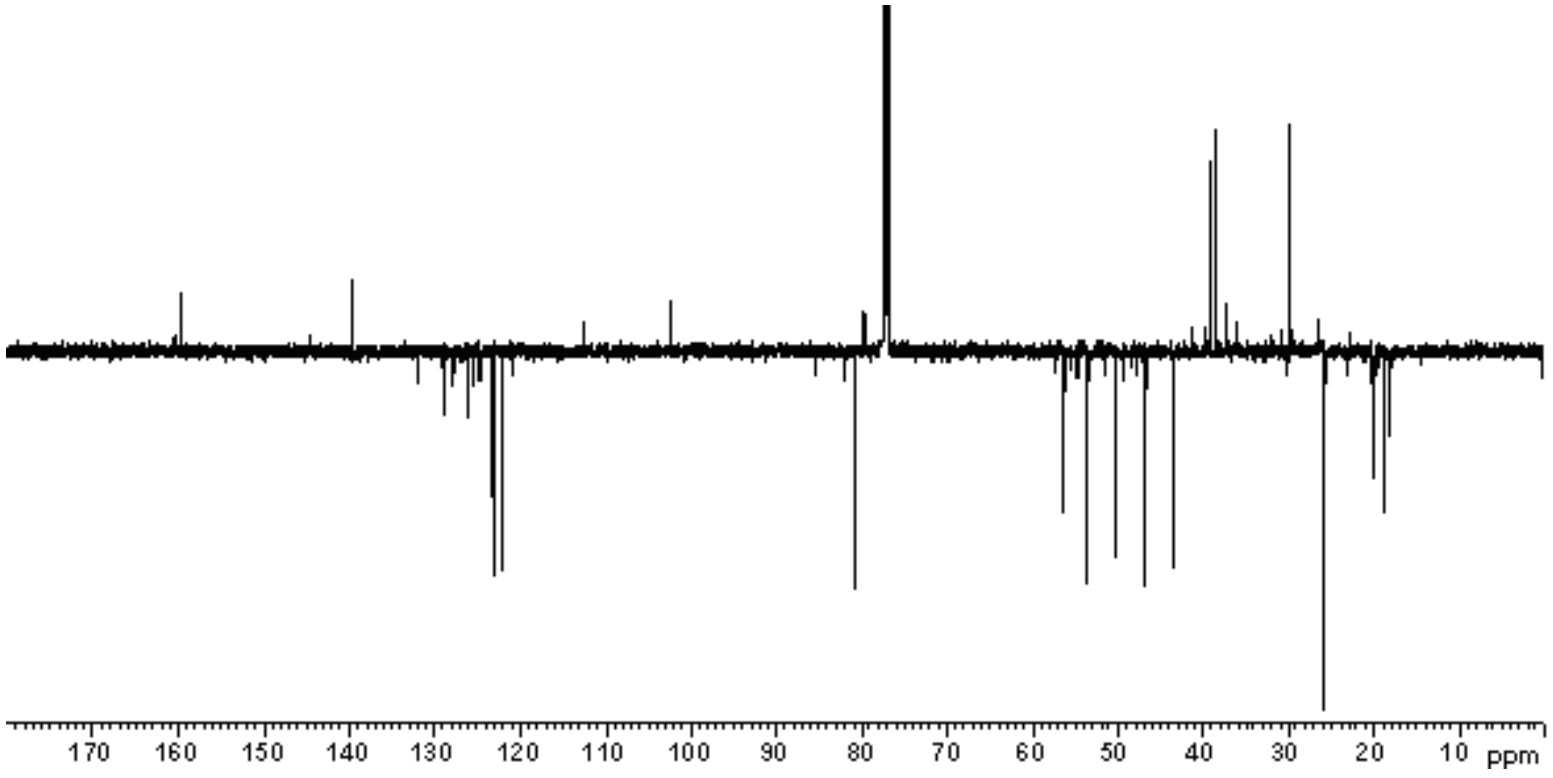

